\title{
FRICTIONAL CONTACT PROBLEM IN DYNAMIC ELECTROELASTICITY
}

\author{
Mostafa Kabbaj and El-H. Essoufi \\ Université Moulay Ismail and Université Hassan Premier, Morocco
}

\begin{abstract}
The dynamic evolution with frictional contact of a electroelastic body is considered. In modelling the contact, the Tresca model is used. We derive a variational formulation for the model in a form of a coupled system involving the displacement and the electric potential fields. We provide existence and uniqueness result. The proof is based on a regularization method, Galerkin method, compactness and lower semicontinuity arguments. Such a result extend the result obtained in [3] where the analysis of friction in dynamic elasticity materials was provided. The novelty of this paper consists in the fact that here we take into account the piezoelectric properties of the materials.
\end{abstract}

\section{Introduction}

Piezoelectricity is the ability of a material to develop an electrical field when it is subjected to a mechanical strain (direct piezoelectric effect) and conversely, develop mechanical strain in response to an applied electric field (converse piezoelectric effect). The coupled mechanical and electrical proprieties of piezoelectric materials make them well suited for use as sensors (which use the direct piezoelectric effect) and actuators (which use the converse piezoelectric effect). A deformable material which presents such a behavior is called a piezoelectric material. Piezoelectric materials are used extensively as switches and actuators in many engineering systems, in radioelectronics, electroacoustics and measuring equipments.

2000 Mathematics Subject Classification. 35J20, 37L65, 46B50, 49J40, 65F22, 74H20, $74 \mathrm{H} 25$.

Key words and phrases. Dynamic electroelasticity, second-order hyperbolic variational inequality, regularization method, Faedo-Galerkin method, compactness method, existence, uniqueness. 
General models for elastic materials with piezoelectric effects can be found in $[16,17,18,21,22]$ and, more recently in $[1,10,20]$. Dynamical contact problems involving elastic or viscoelastic materials without piezoelectric effect have received considerable attention in the mathematical literature, see for instance $[3,12,4,13,14]$ and the references therein. Currently, there is a considerable interest in frictional contact problems involving piezo-electric materials, see for instance $[2,15]$ and the references therein. Indeed, contact is an essential feature of any process in which parts and components of machinery or equipment are in relative motion and friction often accompanies contact, causing wear and tear of contact surfaces and, thereby, reducing the overall performance and reliability of the equipment. However, there exists virtually no mathematical results about contact problems for such materials and there is a need to expand the emerging Mathematical Theory of Contact Mechanics to include the coupling between the mechanical and electrical properties. More recently, some static, quasi-static and dynamical contact problems for piezoelectric body were considered in $[6,7,5]$.

The aim of present paper is to study the process of dynamical frictional contact between a piezoelectric body which is acted upon by volume force and surface tractions, and an obstacle, the so-called foundation, which is electrically nonconducting. So, we assume that the process is dynamic and use the Tresca model for the frictional contact problem. We present weak formulation of the model which consists of a system coupling a variational inequality for the displacement field and a time-dependent variational equation for the electric field and show the existence and uniqueness of the weak solution to model. Such result extend the result obtained in [3] where the analysis of friction in dynamic elasticity materials was provided. Indeed, with respect to the model in [3], the novelty of this paper consists in the fact that here we take into account the piezoelectric properties of the material, which leads to a new and more sophisticate mathematical model.

The rest of the paper is structured as follows. The model of the contact dynamic process of the linear piezoelectric body is presented in Section 2. In Section 3 we list the assumptions on the problem data, derive the variational formulation of the problem and state our main result, Theorem 3.1. The proof of the Theorem 3.1 is presented in Sections 4. It is based on a regularization method, Galerkin method, compactness and lower semicontinuity arguments. In Section 5, we consider a piezo-viscoelastic law with a small viscosity parameter $\eta>0$, and prove that the unique solution of piezo-viscoelastic problem converge when $\eta \rightarrow 0$ to the unique solution of piezo-elastic problem.

\section{Problem Statement}

We consider a body made of a piezoelectric material which occupies in the reference configuration the domain $\Omega \subset \mathbb{R}^{d}, d=1,2,3$, which will be 
supposed bounded with a smooth boundary $\partial \Omega=\Gamma$. Let $\boldsymbol{\nu}$ denote the unit outer normal to $\Gamma$ and let $[0, T]$ be time interval of interest, where $T>0$. We denote by $\boldsymbol{x} \in \Omega \cup \Gamma$ and $t \in[0, T]$ the spatial and the time variable, respectively, and, to simplify the notation, we do not indicate in what follows the dependence of various functions on $\boldsymbol{x}$ and $t$. Everywhere in this paper $i, j, k, l$ run from 1 to $d$, summation over repeated indices is implied and the index that follows a comma represents the partial derivative with respect to the corresponding component of the spatial variable. Moreover, the dot above represents the derivative with respect to the time variable.

Everywhere below we use $\mathbb{S}^{d}$ to denote the space of second order symmetric tensors on $\mathbb{R}^{d}$ while "." and $|\cdot|$ will represent the inner product and the Euclidean norm on $\mathbb{S}^{d}$ and $\mathbb{R}^{d}$, that is

$$
\begin{aligned}
& \boldsymbol{u} \cdot \boldsymbol{v}=u_{i} v_{i}, \quad|\boldsymbol{v}|=(\boldsymbol{v} \cdot \boldsymbol{v})^{1 / 2}, \quad \forall \boldsymbol{u}, \boldsymbol{v} \in \mathbb{R}^{d}, \\
& \boldsymbol{\sigma} \cdot \boldsymbol{\tau}=\sigma_{i j} \tau_{i j}, \quad|\boldsymbol{\tau}|=(\boldsymbol{\tau} \cdot \boldsymbol{\tau})^{1 / 2}, \quad \forall \boldsymbol{\sigma}, \boldsymbol{\tau} \in \mathbb{S}^{d} .
\end{aligned}
$$

We use also the notations $u_{\nu}$ and $\boldsymbol{u}_{\tau}$ for the normal and tangential displacement, that is $u_{\nu}=\boldsymbol{u} \cdot \boldsymbol{\nu}$ and $\boldsymbol{u}_{\tau}=\boldsymbol{u}-u_{\nu} \boldsymbol{\nu}$. We also denote by $\sigma_{\nu}$ and $\boldsymbol{\sigma}_{\tau}$ the normal and tangential stress given by $\sigma_{\nu}=\boldsymbol{\sigma} \boldsymbol{\nu} \cdot \boldsymbol{\nu}, \boldsymbol{\sigma}_{\tau}=\boldsymbol{\sigma} \boldsymbol{\nu}-\sigma_{\nu} \boldsymbol{\nu}$.

In addition, we shall use the following notations:

- $\boldsymbol{u}=\left(u_{i}\right)$ - the displacements field;

- $\varepsilon=\boldsymbol{\varepsilon}(\boldsymbol{u})=\left(\varepsilon_{i j}(\boldsymbol{u})\right)$ - the linearized strain tensor, that is $\varepsilon_{i j}(\boldsymbol{u})=$ $\frac{1}{2}\left(u_{i, j}+u_{j, i}\right)$

- $\boldsymbol{\sigma}=\left(\boldsymbol{\sigma}_{i j}\right)$ - the stress tensor;

- $\varphi$ - the electric potential;

- $\boldsymbol{E}=E(\varphi)=\left(E_{i}(\varphi)\right)$ - the electric field, that is $E_{i}(\varphi)=-\varphi,{ }_{i}$;

- $\boldsymbol{D}=\left(D_{i}\right)$ - the electric displacement field.

Mathematically, a coupled phenomenon of electromechanical interaction can be appropriately described by the system of partial differential equations (see $[20,21,22])$ which includes the equation of motion for continuum media

$$
\rho \ddot{\boldsymbol{u}}-\operatorname{Div} \boldsymbol{\sigma}=\boldsymbol{f}_{0} \quad \text { in } \quad \Omega \times(0, T),
$$

and the static equation for a piezoelectric medium in the acoustic range of frequencies, i.e. the equation of forced electrostatics for dielectrics

$$
\operatorname{div} \boldsymbol{D}=q_{0} \quad \text { in } \quad \Omega \times(0, T),
$$

where $\rho>0$ is the density, $\boldsymbol{f}_{0}$ represents the density of body forces and $q_{0}$ is the volume density of free electric charges. Notice also that Div and div represent the divergence operators for tensor and vector valued functions, that is

$$
\operatorname{Div} \boldsymbol{\sigma}=\left(\sigma_{i j, j}\right), \quad \operatorname{div} \boldsymbol{D}=\left(D_{i, i}\right) .
$$

The constitutive relations for the piezoelectric material are

$$
\boldsymbol{\sigma}=\mathcal{A} \boldsymbol{\varepsilon}(\boldsymbol{u})-\mathcal{E}^{*} \boldsymbol{E}(\varphi) \quad \text { in } \Omega \times(0, T),
$$




$$
\boldsymbol{D}=\mathcal{E} \boldsymbol{\varepsilon}(\boldsymbol{u})+\boldsymbol{\beta} \boldsymbol{E}(\varphi) \quad \text { in } \Omega \times(0, T),
$$

where $\mathcal{A}=\left(a_{i j k l}\right)$ is a (fourth-order) elasticity tensor, $\mathcal{E}=\left(e_{i j k}\right)$ is the (thirdorder) piezoelectric tensor, $\boldsymbol{\beta}=\left(\beta_{i j}\right)$ is the electric permittivity tensor and $\mathcal{E}^{*}$ will be denote the transposite of the tensor $\mathcal{E}$ given by

$$
\mathcal{E} \boldsymbol{\sigma} \cdot \boldsymbol{v}=\boldsymbol{\sigma} \cdot \mathcal{E}^{*} \boldsymbol{v} \quad \forall \boldsymbol{\sigma} \in \mathbb{S}^{d}, \boldsymbol{v} \in \mathbb{R}^{d} .
$$

The relations (2.3)-(2.4) may be written in components as

$$
\sigma_{i j}=a_{i j k l} \varepsilon_{k l}(\boldsymbol{u})-\mathcal{E}_{k i j} E_{k}(\varphi) \text { and } D_{i}=\mathcal{E}_{i j k} \varepsilon_{j k}(\boldsymbol{u})+\beta_{i j} E_{j} .
$$

Notice that (2.3)-(2.4) represents an electro-elastic constitutive law. The equality shows that the mechanical properties of the materials are described by an elastic constitutive relation (for more details, see [3, 8, 9, 10, 20, 12, 11, 12] for elastic and viscoelastic cases) and, moreover, it takes into account the dependence of the stress field on the electric field. Relation (2.4) describes a linear dependence of the electric displacement field on the strain and electric fields; such kind of relations have been frequently considered in the literature, see for instance $[1,10,16,17]$ and the references therein.

Next, to complete the model, we need to prescribe the mechanical and electrical boundary conditions. To this end, we consider first a partition of $\Gamma$ into three disjoints measurable parts $\Gamma_{1}, \Gamma_{2}, \Gamma_{3}$ such that meas $\left(\Gamma_{1}\right)>0$. We assume that the body is clamped on $\Gamma_{1}$ and surfaces traction of density $\boldsymbol{f}_{2}$ act on $\Gamma_{2}$, that is:

$$
\begin{array}{ccc}
\boldsymbol{u}=\mathbf{0} & \text { on } & \Gamma_{1} \times(0, T), \\
\boldsymbol{\sigma} \boldsymbol{\nu}=\boldsymbol{f}_{2} & \text { on } & \Gamma_{2} \times(0, T) .
\end{array}
$$

On $\Gamma_{3}$ the body can arrive in frictional contact with an obstacle, the so-called foundation which is electrically nonconducting and the contact is given by:

$$
\left\{\begin{array}{llll}
\text { (a) } \sigma_{\nu}=-S, S \geq 0, & \text { on } & \Gamma_{3} \times(0, T), \\
\text { (b) } \boldsymbol{\sigma}_{\tau}=-S \mu \frac{\dot{\boldsymbol{u}}_{\tau}}{\left|\dot{\boldsymbol{u}}_{\tau}\right|} & \text { if } \dot{\boldsymbol{u}}_{\tau} \neq 0 & \text { on } & \Gamma_{3} \times(0, T), \\
\text { (c) }\left|\boldsymbol{\sigma}_{\tau}\right| \leq S \mu & \text { if } \dot{\boldsymbol{u}}_{\tau}=0 & \text { on } & \Gamma_{3} \times(0, T) .
\end{array}\right.
$$

These equations represent the Tresca model, i.e. the contact surface is given and we impose a non-positive normal stress $-S$. Relations (2.7) assert that the tangential stress is bounded by the normal stress multiplied by the value of the friction coefficient $\mu$. If such a limit is not attained, sliding does not occur.

Now, to describe the electric boundary conditions we assume that $\Gamma$ is divided into two disjoints measurable parts $\Gamma_{a}$ and $\Gamma_{b}$ such that meas $\left(\Gamma_{a}\right)>0$. 
We also assume, for simplicity, that the electrical potential vanishes on $\Gamma_{a}$ and the electric charge is prescribed on $\Gamma_{b}$. Thus, we use the conditions:

$$
\begin{gathered}
\varphi=0 \quad \text { on } \quad \Gamma_{a} \times(0, T), \\
\boldsymbol{D} \cdot \boldsymbol{\nu}=q_{2} \quad \text { on } \quad \Gamma_{b} \times(0, T),
\end{gathered}
$$

where $q_{2}$ represents the given density of the surface electric charges.

Finally, to complete the model, we prescribe the initial displacement and velocity in $\Omega$, that is

$$
\boldsymbol{u}(0)=\boldsymbol{u}_{0}, \quad \dot{\boldsymbol{u}}(0)=\boldsymbol{v}_{0} \quad \text { in } \quad \Omega,
$$

where $\boldsymbol{u}_{0}$ and $\boldsymbol{v}_{0}$ are given.

Our main goal here is to prove existence and uniqueness of weak solution to the following problem

Problem $P$. Find a displacement field $\boldsymbol{u}: \Omega \times[0, T] \rightarrow \mathbb{R}^{d}$, a stress field $\boldsymbol{\sigma}: \Omega \times[0, T] \rightarrow \mathbb{S}^{d}$, an electric potential field $\varphi: \Omega \times[0, T] \rightarrow \mathbb{R}$ and an electric displacement field $\boldsymbol{D}: \Omega \times[0, T] \rightarrow \mathbb{R}^{d}$ such that (2.1)-(2.10) hold.

In the next section we derive a variational formulations of the problem $P$ and we investigate his solvability. Notice also that the contact frictional conditions (2.7) do not involve the electrical unknowns, that is they represent purely mechanic boundary conditions. An important extension of the result presented in this paper would allow mixed electrical and mechanical condition on the contact surface and will be presented in a forthcoming paper.

\section{Preliminaries and main Results}

Everywhere in what follows we use the usual notation for the $L^{p}$ and Sobolev spaces associated to $\Omega$ and $\Gamma$. Let $X$ be a Banach space, $T$ a positive real number and $1 \leq p \leq \infty$, denotes by $L^{p}(0, T ; X)$ the Banach space of all measurable functions $\boldsymbol{u}:] 0, T\left[\rightarrow X\right.$ such that $t \rightarrow\|\boldsymbol{u}(t)\|_{X}$ is in $L^{p}(0, T)$ with the norm

$$
\|\boldsymbol{u}\|_{p}=\left(\int_{0}^{T}\|\boldsymbol{u}(t)\|_{X}^{p} d t\right)^{\frac{1}{p}}, \text { if } 1 \leq p<\infty
$$

and if $p=\infty$

$$
\|\boldsymbol{u}\|_{\infty}=\operatorname{ess} \sup _{t \in[0, T]}\|\boldsymbol{u}(t)\|_{X} .
$$

For $k=1,2, \ldots$, we also use de classical notations for the $W^{k, p}(0, T ; X)$. Thus, keeping in mind the boundary condition (2.5), we introduce the closed subspace of $\left[H^{1}(\Omega)\right]^{d}$ defined by

$$
V=\left\{\boldsymbol{v}=\left(v_{i}\right) \mid v_{i} \in H^{1}(\Omega), \boldsymbol{v}=\mathbf{0} \text { on } \Gamma_{1}\right\} .
$$

For the stress filed we use the spaces

$$
Q=\left\{\boldsymbol{\tau}=\left(\tau_{i j}\right) \mid \tau_{i j}=\tau_{j i} \in L^{2}(\Omega)\right\},
$$




$$
Q_{1}=\left\{\boldsymbol{\tau} \in Q \mid \tau_{i j, j} \in L^{2}(\Omega)\right\} .
$$

Recall that the spaces $Q$ and $Q_{1}$ are real Hilbert spaces endowed with the inner products

$$
\begin{aligned}
& (\boldsymbol{\sigma}, \boldsymbol{\tau})_{Q}=\int_{\Omega} \sigma_{i j} \tau_{i j} d x, \\
& (\boldsymbol{\sigma}, \boldsymbol{\tau})_{Q_{1}}=(\boldsymbol{\sigma}, \boldsymbol{\tau})_{Q}+(\operatorname{Div} \boldsymbol{\sigma}, \operatorname{Div} \boldsymbol{\tau})_{L^{2}(\Omega)^{d}}
\end{aligned}
$$

and the associated norms $\|\cdot\|_{Q}$ and $\|\cdot\|_{Q_{1}}$, respectively. Over the space $V$ we use the inner product

$$
(\boldsymbol{u}, \boldsymbol{v})_{V}=(\varepsilon(\boldsymbol{u}), \boldsymbol{\varepsilon}(\boldsymbol{v}))_{Q}
$$

and let $\|\cdot\|$ be the associated norm. Since meas $\left(\Gamma_{1}\right)>0$, it follows from Korn's inequality (see e.g. [19] p.79) that $\|\cdot\|$ and $\|\cdot\|_{\left[H^{1}(\Omega)\right]^{d}}$ are equivalent norms on $V$, thus $\left(V,(\cdot, \cdot)_{V}\right)$ is a real Hilbert space. Let us denote $H:=\left[L^{2}(\Omega)\right]^{d}$ endowed with the inner product

$$
(\boldsymbol{u}, \boldsymbol{v}):=\int_{\Omega} \rho \boldsymbol{u} \cdot \boldsymbol{v} d x, \forall \boldsymbol{u}, \boldsymbol{v} \in H,
$$

which generates an equivalent norm (by using (3.10)) denoted by $|\cdot|$. Since $V$ is dense in $H$, we identify $H$ and $H^{\prime}$ and we write $V \subset H \equiv H^{\prime} \subset V^{\prime}$.

Next, for the electric potential field and the electric displacement field we use respectively the functional spaces

$$
W=\left\{\psi \in H^{1}(\Omega) \mid \psi=0 \text { on } \Gamma_{a}\right\}
$$

and

$$
\mathcal{W}=\left\{\boldsymbol{D}=\left(D_{i}\right) \mid D_{i} \in L^{2}(\Omega), D_{i, i} \in L^{2}(\Omega)\right\} .
$$

The spaces $W$ and $\mathcal{W}$ are real Hilbert spaces with the inner products

$$
\begin{gathered}
(\varphi, \psi)_{W}=(\varphi, \psi)_{H^{1}(\Omega)}, \\
(\boldsymbol{D}, \boldsymbol{E})_{\mathcal{W}}=(\boldsymbol{D}, \boldsymbol{E})_{L^{2}(\Omega)^{d}}+(\operatorname{div} \boldsymbol{D}, \operatorname{div} \boldsymbol{E})_{L^{2}(\Omega)}
\end{gathered}
$$

and the associated norms $\|\cdot\|_{W}$ and $\|\cdot\|_{\mathcal{W}}$ respectively and since $W$ is dense in $L^{2}(\Omega)$, we write $W \subset L^{2}(\Omega) \subset W^{\prime}$.

In the study of Problem $P$ we assume that the elasticity tensor $\mathcal{A}$, the piezoelectric tensor $\mathcal{E}$ and the electric permittivity tensor $\boldsymbol{\beta}$ satisfy:

$$
\begin{aligned}
& \text { (a) } \mathcal{A}=\left(a_{i j k l}\right): \Omega \times \mathbb{S}^{d} \rightarrow \mathbb{S}^{d} . \\
& \text { (b) } a_{i j k l}=a_{k l i j}=a_{j i k l} \in L^{\infty}(\Omega) . \\
& \text { (c) } \exists m_{\mathcal{A}}>0 \text { such that } a_{i j k l} \varepsilon_{i j} \varepsilon_{k l} \geq m_{\mathcal{A}}|\varepsilon|^{2} \\
& \forall \varepsilon \in \mathbb{S}^{d}, \text { a.e. in } \Omega .
\end{aligned}
$$

$$
\left\{\begin{array}{l}
\text { (a) } \mathcal{E}=\left(e_{i j k}\right): \Omega \times \mathbb{S}^{d} \rightarrow \mathbb{R}^{d} . \\
\text { (b) } e_{i j k}=e_{i k j} \in L^{\infty}(\Omega) .
\end{array}\right.
$$




$$
\left\{\begin{array}{l}
\text { (a) } \boldsymbol{\beta}=\left(\beta_{i j}\right): \Omega \times \mathbb{R}^{d} \rightarrow \mathbb{R}^{d} . \\
\text { (b) } \beta_{i j}=\beta_{j i} \in L^{\infty}(\Omega) . \\
\text { (c) } \exists m_{\beta}>0, \text { such that } \beta_{i j} E_{i} E_{j} \geq m_{\beta}|\boldsymbol{E}|^{2} \\
\forall \boldsymbol{E} \in \mathbb{R}^{d} \text {, a.e. in } \Omega .
\end{array}\right.
$$

We denote by $a: V \times V \longrightarrow \mathbb{R}$ and $b: W \times W \longrightarrow \mathbb{R}$ the following bilinear and symmetric applications

$$
a(\boldsymbol{u}, \boldsymbol{v}):=(\mathcal{A} \varepsilon(\boldsymbol{u}), \boldsymbol{\varepsilon}(\boldsymbol{v}))_{Q}, \quad b(\varphi, \psi):=(\beta \nabla \varphi, \nabla \psi)_{L^{2}(\Omega)^{d}}
$$

We also denote by $e: V \times W \longrightarrow \mathbb{R}$ the following bilinear application

$$
e(\boldsymbol{v}, \psi):=(\mathcal{E} \varepsilon(\boldsymbol{v}), \nabla \psi)_{L^{2}(\Omega)^{d}}=\left(\mathcal{E}^{*} \nabla \psi, \varepsilon(\boldsymbol{v})\right)_{Q}
$$

Then from (3.1)-(3.4) we find $M_{\mathcal{A}}>0, M_{\mathcal{E}}>0$ and $M_{\beta}>0$ such that

$$
\begin{gathered}
|a(\boldsymbol{u}, \boldsymbol{v})| \leq M_{\mathcal{A}}\|\boldsymbol{u}\|\|\boldsymbol{v}\|, \quad \forall \boldsymbol{u}, \boldsymbol{v} \in V, \\
|b(\varphi, \psi)| \leq M_{\beta}\|\varphi\|_{W}\|\psi\|_{W}, \quad \forall \varphi, \psi \in W, \\
|e(\boldsymbol{v}, \psi)| \leq M_{\mathcal{E}}\|\boldsymbol{v}\|\|\psi\|_{W}, \quad \forall \boldsymbol{v} \in V, \forall \psi \in W .
\end{gathered}
$$

From (3.2), (3.4), Korn inequality and Poincaré inequality we deduce that there exists $m_{\mathcal{A}}>0$ and $m_{b}>0$ such that

$$
a(\boldsymbol{v}, \boldsymbol{v}) \geq m_{\mathcal{A}}\|\boldsymbol{v}\|^{2}, \quad b(\psi, \psi) \geq m_{b}\|\psi\|_{W}^{2}, \quad \forall \boldsymbol{v} \in V, \forall \psi \in W .
$$

The friction coefficient $\mu: \Gamma_{3} \mapsto \mathbb{R}_{+}$is assumed to satisfy

$$
\mu(s) \geq 0 \text { for all } s \in \Gamma_{3} \text { and } \mu \in L^{\infty}\left(\Gamma_{3}\right) .
$$

For the density and the normal stress we suppose that the following conditions are satisfied

$\rho \in L^{\infty}(\Omega)$ and there exists $\rho_{0}$ such that $0<\rho_{0} \leq \rho(x)$, a.e. $x \in \Omega$

and

$$
S(s) \geq 0 \text { for all } s \in \Gamma_{3} \text { and } S \in L^{\infty}\left(\Gamma_{3}\right) .
$$

The body forces and the surface tractions have the regularity

$$
\boldsymbol{f}_{0} \in W^{2,2}\left(0, T ; L^{2}(\Omega)^{d}\right), \quad \boldsymbol{f}_{2} \in W^{2,2}\left(0, T ; L^{2}\left(\Gamma_{2}\right)^{d}\right) .
$$

The volume and surface densities of the electric charges satisfy

$$
q_{0} \in W^{2,2}\left(0, T ; L^{2}(\Omega)\right), q_{2} \in W^{2,2}\left(0, T ; L^{2}\left(\Gamma_{b}\right)\right) .
$$

The initial conditions satisfy

$$
\boldsymbol{u}_{0}, \boldsymbol{v}_{0} \in V \text {. }
$$


Next, using Riesz's representation theorem, we define the elements $\boldsymbol{f}(t) \in V^{\prime}$ and $q(t) \in W^{\prime}$ respectively by

$$
\begin{gathered}
\langle\boldsymbol{f}(t), \boldsymbol{v}\rangle_{V^{\prime}, V}=\int_{\Omega} \boldsymbol{f}_{0}(t) \cdot \boldsymbol{v} d x+\int_{\Gamma_{2}} \boldsymbol{f}_{2}(s, t) \cdot \boldsymbol{v}(s) d s-\int_{\Gamma_{3}} S(s) v_{\nu}(s) d s, \\
\langle q(t), \psi\rangle_{W^{\prime}, W}=\int_{\Omega} q_{0}(t) \psi d x-\int_{\Gamma_{b}} q_{2}(t) \psi d a
\end{gathered}
$$

for all $\boldsymbol{v} \in V, \psi \in W$ and $t \in[0, T]$. Keeping in mind assumptions (3.12)(3.13), it follows that the integrals in (3.15)-(3.16) are well-defined and we have the following regularities

$$
\boldsymbol{f} \in W^{2,2}\left(0, T ; V^{\prime}\right) \text { and } q \in W^{2,2}\left(0, T ; W^{\prime}\right)
$$

Having in mind (3.6)-(3.8) and (3.13)-(3.14) the variational problem

$$
b\left(\varphi_{0}, \psi\right)=e\left(\boldsymbol{u}_{0}, \psi\right)+\langle q(0), \psi\rangle_{W^{\prime}, W} \forall \psi \in W
$$

has a unique solution $\varphi_{0} \in W$. Hence, we can define

$$
\boldsymbol{\sigma}(0)=\boldsymbol{\sigma}_{0}=\mathcal{A} \boldsymbol{\varepsilon}\left(\boldsymbol{u}_{0}\right)-\mathcal{E} \nabla \varphi_{0} .
$$

In addition we suppose that

$$
\text { Div } \boldsymbol{\sigma}_{0} \in H \text { and } \boldsymbol{\sigma}_{0} \boldsymbol{\nu}=\boldsymbol{f}_{2}(0) \text { on } \Gamma_{2},
$$

and we assume that at initial time $t=0$ there is no frictional process on $\Gamma_{3}$ :

$$
\boldsymbol{\sigma}_{0 \tau}=0, \boldsymbol{v}_{0 \tau}=0 \text { on } \Gamma_{3} .
$$

Relations (3.18) give the regularity of the initial data and ensure the compatibility between the initial data and the boundary conditions.

Finally, we define the functional $j: V \mapsto \mathbb{R}$ as follows:

$$
j(\boldsymbol{v}):=\int_{\Gamma_{3}} S(s) \mu(s)\left|\boldsymbol{v}_{\tau}(s)\right| d s, \forall \boldsymbol{v} \in V .
$$

Using integration by parts, it is straightforward to see that if $(\boldsymbol{u}, \boldsymbol{\sigma}, \boldsymbol{\varphi}, \boldsymbol{D})$ are sufficiently regular functions satisfying (2.1)-(2.10) then, for all $t \in[0, T]$, we have

$$
\begin{gathered}
(\ddot{\boldsymbol{u}}(t), \boldsymbol{w})+(\boldsymbol{\sigma}(t), \boldsymbol{\varepsilon}(\boldsymbol{w}))_{Q}=\int_{\Gamma_{3}} \boldsymbol{\sigma}_{\tau}(t) \cdot \boldsymbol{w}_{\tau} d s+\langle\boldsymbol{f}(t), \boldsymbol{w}\rangle_{V^{\prime}, V}, \forall \boldsymbol{w} \in V, \\
(\boldsymbol{D}(t), \nabla \psi)_{L^{2}(\Omega)^{d}}+\langle q(t), \psi\rangle_{W^{\prime}, W}=0, \forall \psi \in W
\end{gathered}
$$

Keeping in mind (2.7), we find

$$
\boldsymbol{\sigma}_{\tau} \cdot\left(\boldsymbol{v}_{\tau}-\dot{\boldsymbol{u}}_{\tau}\right)+S \mu(s)\left(\left|\boldsymbol{v}_{\tau}\right|-\left|\dot{\boldsymbol{u}}_{\tau}\right|\right) \geq 0 \text {, a.e. on } \Gamma_{3} \times[0, T] .
$$

Therefore, taking $\boldsymbol{w}=\boldsymbol{v}-\dot{\boldsymbol{u}}(t)$ in (3.21), we have:

$$
\begin{gathered}
(\ddot{\boldsymbol{u}}(t), \boldsymbol{v}-\dot{\boldsymbol{u}}(t))+\left(\boldsymbol{\sigma}(t), \boldsymbol{\varepsilon}(\boldsymbol{v})-\boldsymbol{\varepsilon}(\dot{\boldsymbol{u}}(t))_{Q}+j(\boldsymbol{v})-j(\dot{\boldsymbol{u}}(t))\right. \\
\geq\langle\boldsymbol{f}(t), \boldsymbol{v}-\dot{\boldsymbol{u}}(t)\rangle_{V^{\prime}, V}, \forall \boldsymbol{v} \in V, t \in[0, T]
\end{gathered}
$$




$$
(\boldsymbol{D}(t), \nabla \psi)_{L^{2}(\Omega)^{d}}+\langle q(t), \psi\rangle_{W^{\prime}, W}=0, \forall \psi \in W, t \in[0, T] .
$$

Here and below we use $\nabla \psi$ to denote the gradient of the scalar function $\psi \in W$, that is $\nabla \psi=\left(\psi,{ }_{i}\right)$. We substitute now (2.3) in (3.23), (2.4) in (3.24), use the notation $\boldsymbol{E}=-\nabla \varphi$ and the initial condition (2.10) to derive the following variational formulation of problem $P$, in the terms of displacement and electric potential fields.

Problem $P_{V}$. Find the displacement field $\boldsymbol{u}:[0, T] \rightarrow V$ and the electric potential $\varphi:[0, T] \rightarrow W$ such that

$$
\begin{gathered}
(\ddot{\boldsymbol{u}}(t), \boldsymbol{v}-\dot{\boldsymbol{u}}(t))+a(\boldsymbol{u}(t), \boldsymbol{v}-\dot{\boldsymbol{u}}(t))+e(\boldsymbol{v}-\dot{\boldsymbol{u}}(t), \varphi(t)) \\
+j(\boldsymbol{v})-j(\dot{\boldsymbol{u}}(t)) \geq\langle\boldsymbol{f}(t), \boldsymbol{v}-\dot{\boldsymbol{u}}(t)\rangle_{V^{\prime}, V} \quad \forall \boldsymbol{v} \in V, t \in[0, T], \\
b(\varphi(t), \psi)-e(\boldsymbol{u}(t)), \psi)=\langle q(t), \psi\rangle_{W^{\prime}, W} \quad \forall \psi \in W, t \in[0, T], \\
\boldsymbol{u}(0)=\boldsymbol{u}_{0}, \quad \dot{\boldsymbol{u}}(0)=\boldsymbol{v}_{0} .
\end{gathered}
$$

The main result of this section is the following:

Theorem 3.1. Assume that (3.2)-(3.4), (3.9)-(3.14) and (3.18)-(3.19) hold. Then there exists a unique solution $(\boldsymbol{u}, \varphi)$ of Problem $P_{V}$ such that

$$
\boldsymbol{u} \in W^{1, \infty}(0, T ; V) \cap W^{2, \infty}(0, T ; H), \varphi \in W^{1, \infty}(0, T ; W) .
$$

A "quadruple" of functions $(\boldsymbol{u}, \boldsymbol{\sigma}, \varphi, \boldsymbol{D})$ which satisfy (2.3)-(2.4), (3.25)(3.27) is called a weak solution of the piezoelectric contact problem $P$. We conclude by Theorem 3.1 that, under the assumptions (3.2)-(3.4), (3.9)-(3.14) and (3.18)-(3.19), there exists a unique weak solution of Problem $P$.

\section{Proof of Theorem 3.1}

The proof of Theorem 3.1 will be carried out in several steps. First, we prove the uniqueness result.

UNIQUENESS. Let consider two solutions $\left(\boldsymbol{u}_{1}, \varphi_{1}\right),\left(\boldsymbol{u}_{2}, \varphi_{2}\right)$ to problem $P_{V}$ with the regularity $(3.28)$ and write $\boldsymbol{w}(t)=\boldsymbol{u}_{2}(t)-\boldsymbol{u}_{1}(t)$ and $\phi(t)=$ $\varphi_{2}(t)-\varphi_{1}(t)$. If we write the variational inequality (3.25) successively for $\left(\boldsymbol{u}_{1}, \varphi_{1}\right)$ and $\left(\boldsymbol{u}_{2}, \varphi_{2}\right)$, taking $\boldsymbol{v}=\dot{\boldsymbol{u}}_{2}(t)$ in the first inequality and $\boldsymbol{v}=\dot{\boldsymbol{u}}_{1}(t)$ in the second one, and add the resulting inequalities, we obtain

$$
(\ddot{\boldsymbol{w}}(t), \dot{\boldsymbol{w}}(t))+a(\boldsymbol{w}(t), \dot{\boldsymbol{w}}(t))+e(\dot{\boldsymbol{w}}(t), \phi(t)) \leq 0, \forall t \in(0, T) .
$$

We can easily deduce from variational equality (3.26) that

$$
e(\boldsymbol{w}(t), \psi)=b(\phi(t), \psi), \forall t \in(0, T), \forall \psi \in W,
$$

which implies that

$$
e(\dot{\boldsymbol{w}}(t), \phi(t))=b(\dot{\phi}(t), \phi(t)), \forall t \in(0, T) .
$$


Then, from (4.1) and (4.3) we have

$$
\frac{1}{2} \frac{d}{d t}\left(|\dot{\boldsymbol{w}}(t)|^{2}+a(\boldsymbol{w}(t), \boldsymbol{w}(t))+b(\phi(t), \phi(t))\right) \leq 0, \forall t \in(0, T) .
$$

Using $\boldsymbol{w}(0)=\mathbf{0},(4.2)$ with $\psi=\phi(0)$ and $t=0$, and coercivity of the bilinear form $b(\cdot, \cdot)$ we get $\phi(0)=0$. Since $\boldsymbol{w}(0)=\dot{\boldsymbol{w}}(0)=\mathbf{0}, \phi(0)=0$, we deduce from the above inequality that $\boldsymbol{w}=\mathbf{0}$ and $\phi=0, \forall t \in[0, T]$.

Existence. Firstly, by using Riesz's representation theorem we find the operators: $B: W \rightarrow W^{\prime}, \Theta: V \rightarrow W^{\prime}$ and $\Theta^{*}: W \rightarrow V^{\prime}$ defined by:

$$
\begin{gathered}
\langle B \varphi, \psi\rangle_{W^{\prime} W}=b(\varphi, \psi), \forall \psi \in W, \\
\langle\Theta \boldsymbol{v}, \psi\rangle_{W^{\prime}, W}=(\mathcal{E} \varepsilon(\boldsymbol{v}), \nabla \psi)_{L^{2}(\Omega)^{d}}=e(\boldsymbol{v}, \psi) \quad \forall \psi \in W, \\
\left\langle\Theta^{*} \varphi, \boldsymbol{v}\right\rangle_{V^{\prime}, V}=\left(\mathcal{E}^{*} \nabla \varphi, \boldsymbol{\varepsilon}(\boldsymbol{v})\right)_{Q}=e(\boldsymbol{v}, \varphi) \quad \forall \boldsymbol{v} \in V
\end{gathered}
$$

where $\Theta^{*}$ is the adjoint of $\Theta$. Having in mind (3.4) we observe that $B$ is positive definite-adjoint operator.

Now, we prove the following equivalence result.

Lemma 4.1. The couple $(\boldsymbol{u}, \varphi)$ is a solution to problem $P_{V}$ if only if $(\ddot{\boldsymbol{u}}(t), \boldsymbol{v}-\dot{\boldsymbol{u}}(t))+a(\boldsymbol{u}(t), \boldsymbol{v}-\dot{\boldsymbol{u}}(t))+\left\langle\Theta^{*} B^{-1} \Theta \boldsymbol{u}(t), \boldsymbol{v}-\dot{\boldsymbol{u}}(t)\right\rangle_{V^{\prime}, V}+j(\boldsymbol{v})-j(\dot{\boldsymbol{u}}(t))$

$$
\begin{gathered}
\geq\left\langle\boldsymbol{f}(t)-\Theta^{*} B^{-1} q(t), \boldsymbol{v}-\dot{\boldsymbol{u}}(t)\right\rangle_{V^{\prime}, V}, \forall \boldsymbol{v} \in V, \forall t \in[0, T], \\
\boldsymbol{u}(0)=\boldsymbol{u}_{0}, \quad \dot{\boldsymbol{u}}(0)=\boldsymbol{v}_{0}, \\
\varphi(t)=B^{-1} \Theta \boldsymbol{u}(t)+B^{-1} q(t), \forall t \in[0, T] .
\end{gathered}
$$

Proof. Let $(\boldsymbol{u}, \varphi)$ be a solution of $P_{V}$. We solve the equation (3.26) on $\boldsymbol{u}(t)$. To this end, let $\boldsymbol{u} \in C(0, T ; V)$ and find $\varphi:[0, T] \rightarrow W$ such that

$$
b(\varphi(t), \psi)=e(\boldsymbol{u}(t), \psi)+\langle q(t), \psi\rangle_{W^{\prime}, W}, \forall \psi \in W, \forall t \in[0, T] .
$$

It follows from $(3.4)$ that the bilinear form $b(\cdot, \cdot)$ is continuous, symmetric and coercive on $W$. Moreover, keeping in mind the assumption (3.3) on the piezoelectric tensor $\mathcal{E}$ it follows that the linear form defined on $W$ by:

$$
\psi \mapsto(\mathcal{E} \varepsilon(\boldsymbol{u}(t)), \nabla \psi)_{L^{2}(\Omega)^{d}}+\langle q(t), \psi\rangle_{W^{\prime}, W}
$$

is continuous on $W$ and belong to $C\left(0, T ; W^{\prime}\right)$. Using the Lax-Milgram theorem, we conclude that the equation (4.11) has unique solution $\varphi \in C(0, T ; W)$.

Thus, using the equations (4.5), (4.6) and (4.7), we write equation (3.26) in a explicit form i.e. equation (4.10) and then equation (3.25) is equivalent to (4.8), thus the problem $P_{V}$ is now equivalent to: find $\boldsymbol{u}:[0, T] \rightarrow V$ and $\varphi:[0, T] \rightarrow W$ such that (4.8), (4.9) and (4.10) are satisfied. 
Now, to show the global existence of solution $\boldsymbol{u}$ of the problem (4.8) - (4.9). To this end, we will use the Faedo-Galerkin's and compactness methods. Let us consider $\left(\phi_{i}\right)_{i \in \mathbb{N}} \in V$ a sequence of linear independent functions such that $V=\bar{\cup}_{m=1}^{\infty} V^{m}$ where $V^{m}=\operatorname{Span}\left\{\phi_{1}, \phi_{2}, \ldots, \phi_{m}\right\}$. We shall suppose that $V^{m}$ is chosen such that for $m$ large enough we have $\boldsymbol{u}_{0}$ and $\boldsymbol{v}_{0}$ belong to $V^{m}$.

We also consider, for all $\epsilon>0$, the family of convex and differentiable functions $\psi_{\epsilon}: \mathbb{R}^{d} \rightarrow \mathbb{R}$ given by

$$
\psi_{\epsilon}(\boldsymbol{v})=\sqrt{|\boldsymbol{v}|^{2}+\epsilon^{2}}-\epsilon, \boldsymbol{v} \in \mathbb{R}^{d} .
$$

It is easy to show that such a family of functions satisfies:

$$
\begin{gathered}
0 \leq \psi_{\epsilon}(\boldsymbol{v}) \leq|\boldsymbol{v}|, \forall \boldsymbol{v} \in \mathbb{R}^{d}, \\
\left|\psi_{\epsilon}(\boldsymbol{v})-\right| \boldsymbol{v}|| \leq \epsilon, \forall \boldsymbol{v} \in \mathbb{R}^{d},
\end{gathered}
$$

and

$$
\psi_{\epsilon}^{\prime}(\boldsymbol{v})(\boldsymbol{w})=\frac{\boldsymbol{v} \cdot \boldsymbol{w}}{\sqrt{|\boldsymbol{v}|^{2}+\epsilon^{2}}}, \forall \boldsymbol{v}, \boldsymbol{w} \in \mathbb{R}^{d} .
$$

From equation (4.14), we have

$$
\left|\psi_{\epsilon}^{\prime}(\boldsymbol{v})(\boldsymbol{w})\right| \leq|\boldsymbol{w}|, \forall \boldsymbol{v}, \boldsymbol{w} \in \mathbb{R}^{d},
$$

We then define a family of regularized frictional functional $j_{\epsilon}: V \rightarrow \mathbb{R}$ by

$$
j_{\epsilon}(\boldsymbol{v})=\int_{\Gamma_{3}} S(s) \mu(s) \psi_{\epsilon}\left(\boldsymbol{v}_{\tau}(s)\right) d s, \forall \boldsymbol{v} \in V .
$$

The functionals $j_{\epsilon}$ are Gâteaux-differentiable with respect to $\boldsymbol{v}$ and represent an approximation of $j$, i.e. there exists a constant $C$ such that

$$
\left|j_{\epsilon}(\boldsymbol{v})-j(\boldsymbol{v})\right| \leq C \epsilon, \forall \boldsymbol{v} \in V .
$$

We denote by $J_{\epsilon}: V \rightarrow V^{\prime}$ the derivative of $j_{\epsilon}$ given by

$$
<J_{\epsilon}(\boldsymbol{v}), \boldsymbol{w}>_{V^{\prime}, V}=\int_{\Gamma_{3}} S(s) \mu(s) \psi_{\epsilon}^{\prime}\left(\boldsymbol{v}_{\tau}(s)\left(\boldsymbol{w}_{\tau}(s)\right) d s, \forall \boldsymbol{v}, \boldsymbol{w} \in V .\right.
$$

Now, we define the regularized problem associated to (4.8)-(4.9) in finitedimensional spaces: find $\boldsymbol{u}_{\epsilon}^{m}:[0, T] \rightarrow V^{m}$ such that:

$$
\begin{gathered}
\left(\ddot{\boldsymbol{u}}_{\epsilon}^{m}(t), \boldsymbol{v}\right)+a\left(\boldsymbol{u}_{\epsilon}^{m}(t), \boldsymbol{v}\right)+\left\langle\Theta^{*} B^{-1} \Theta \boldsymbol{u}_{\epsilon}^{m}(t), \boldsymbol{v}\right\rangle_{V^{\prime}, V}+\left\langle J_{\epsilon}\left(\dot{\boldsymbol{u}}_{\epsilon}^{m}(t), \boldsymbol{v}\right\rangle_{V^{\prime}, V}\right. \\
=\left\langle\boldsymbol{f}(t)-\Theta^{*} B^{-1} q(t), \boldsymbol{v}\right\rangle_{V^{\prime}, V}, \forall \boldsymbol{v} \in V^{m}, \forall t \in[0, T] . \\
\boldsymbol{u}_{\epsilon}^{m}(0)=\boldsymbol{u}_{0}, \quad \dot{\boldsymbol{u}}_{\epsilon}^{m}(0)=\boldsymbol{v}_{0},
\end{gathered}
$$

Since $\boldsymbol{v} \rightarrow J_{\epsilon}(\boldsymbol{v})$ is Lipschitz continuous on $V^{m}$ we deduce that for all $\epsilon>0$ and $m \in \mathbb{N}$ the problem(4.19)-(4.20) has an unique global solution $\boldsymbol{u}_{\epsilon}^{m} \in$ $C^{2}\left(0, T ; V^{m}\right)$.

In the next, we provide convergence result involving the sequences $\left\{\boldsymbol{u}_{\epsilon}^{m}\right\}$. To this end, we need a priori estimates to pass to the limit as $\epsilon \rightarrow 0$ and 
$m \rightarrow \infty$. To simplify the exposition, we omit the indices $m$ and $\epsilon$ whenever it is unambiguous. We have the following estimates:

Estimate I. Since $\left\langle J_{\epsilon}(\boldsymbol{v}), \boldsymbol{v}\right\rangle_{V^{\prime}, V} \geq 0$ for all $\boldsymbol{v} \in V$, therefore if we take $\boldsymbol{v}=\dot{\boldsymbol{u}}(t)$ in $(4.19)$, we obtain

(4.21) $\frac{1}{2} \frac{d}{d t}\left(|\dot{\boldsymbol{u}}(t)|^{2}+a(\boldsymbol{u}(t), \boldsymbol{u}(t))+\left\langle\Theta^{*} B^{-1} \Theta \boldsymbol{u}, \boldsymbol{u}\right\rangle_{V^{\prime}, V}\right) \leq\langle\boldsymbol{h}(t), \dot{\boldsymbol{u}}(t)\rangle_{V^{\prime}, V}$

where $\boldsymbol{h}(t)=\boldsymbol{f}(t)-\Theta^{*} B^{-1} q(t)$. We observe that there exists $\alpha>0$ such that

$$
\left\langle\Theta^{*} B^{-1} \Theta \boldsymbol{u}, \boldsymbol{u}\right\rangle_{V^{\prime}, V}=\left\langle\Theta \boldsymbol{u}, B^{-1} \Theta \boldsymbol{u}\right\rangle_{W^{\prime} \times W} \geq \alpha\|\Theta \boldsymbol{u}\|_{W^{\prime}}^{2}
$$

If we integrate this inequality over $(0, t)$ and we use the coercivity of the form $a(\cdot, \cdot)$ and $(4.22)$ then we find

$$
\begin{aligned}
|\dot{\boldsymbol{u}}(t)|^{2} & +m_{\mathcal{A}}\|\boldsymbol{u}(t)\|^{2}+\alpha\|\Theta \boldsymbol{u}(t)\|_{W^{\prime}}^{2} \leq\left|\boldsymbol{v}_{0}\right|^{2}+a\left(\boldsymbol{u}_{0}, \boldsymbol{u}_{0}\right)+\left\langle\Theta^{*} B^{-1} \Theta \boldsymbol{u}_{0}, \boldsymbol{u}_{0}\right\rangle_{V^{\prime}, V} \\
& +2\langle\boldsymbol{h}(t), \boldsymbol{u}(t)\rangle_{V^{\prime}, V}-2\left\langle\boldsymbol{h}(0), \boldsymbol{u}_{0}\right\rangle_{V^{\prime}, V}-2 \int_{0}^{t}\langle\dot{\boldsymbol{h}}(\theta), \boldsymbol{u}(\theta)\rangle_{V^{\prime}, V} d \theta
\end{aligned}
$$

Now, by using the assumptions on the data and Gronwall's inequality, it is straightforward to obtain the following estimates

$$
\left\{\begin{array}{l}
\left\{\boldsymbol{u}_{\epsilon}^{m}\right\}_{m, \epsilon} \text { is bounded in } L^{\infty}(0, T ; V) \\
\left\{\dot{\boldsymbol{u}}_{\epsilon}^{m}\right\}_{m, \epsilon} \text { is bounded in } L^{\infty}(0, T ; H)
\end{array}\right.
$$

Estimate II. Differentiating the equation (4.19) with respect to $t$ and replacing $\boldsymbol{v}$ by $\ddot{\boldsymbol{u}}(t)$ we have

$$
\begin{aligned}
(\dddot{\boldsymbol{u}}(t), \ddot{\boldsymbol{u}}(t))+a(\dot{\boldsymbol{u}}(t), & \ddot{\boldsymbol{u}}(t))+\left\langle\Theta^{*} B^{-1} \Theta \dot{\boldsymbol{u}}(t), \ddot{\boldsymbol{u}}(t)\right\rangle_{V^{\prime}, V} \\
& +\left\langle\frac{d}{d t} J_{\epsilon}(\dot{\boldsymbol{u}}(t)), \ddot{\boldsymbol{u}}(t)\right\rangle_{V^{\prime}, V}=\langle\dot{\boldsymbol{h}}(t), \ddot{\boldsymbol{u}}(t)\rangle_{V^{\prime}, V} .
\end{aligned}
$$

Since

$$
\begin{aligned}
& \left\langle\frac{d}{d t} J_{\epsilon}(\dot{\boldsymbol{u}}(t)), \ddot{\boldsymbol{u}}(t)\right\rangle_{V^{\prime}, V} \\
& \quad=\int_{\Gamma_{3}} \frac{S(s) \mu(s)}{\left(\left|\dot{\boldsymbol{u}}_{\tau}\right|^{2}+\epsilon^{2}\right)^{\frac{3}{2}}}\left[\left|\ddot{\boldsymbol{u}}_{\tau}\right|^{2}\left|\dot{\boldsymbol{u}}_{\tau}\right|^{2}-\left(\dot{\boldsymbol{u}}_{\tau} \cdot \ddot{\boldsymbol{u}}_{\tau}\right)^{2}+\epsilon^{2}\left|\ddot{\boldsymbol{u}}_{\tau}\right|^{2}\right] d s \geq 0,
\end{aligned}
$$

the equation (4.24) implies that

$(4.25)$

$$
\frac{1}{2} \frac{d}{d t}\left(|\ddot{\boldsymbol{u}}(t)|^{2}+a(\dot{\boldsymbol{u}}(t), \dot{\boldsymbol{u}}(t))+\left\langle\Theta^{*} B^{-1} \Theta \dot{\boldsymbol{u}}(t), \dot{\boldsymbol{u}}(t)\right\rangle_{V^{\prime}, V}\right) \leq\langle\dot{\boldsymbol{h}}(t), \ddot{\boldsymbol{u}}(t)\rangle_{V^{\prime}, V}
$$


Now, using coercivity of the form $a(\cdot, \cdot)$ together with (4.22) and integrating over $(0, t), 0 \leq t \leq T$, we get

$$
\begin{aligned}
|\ddot{\boldsymbol{u}}(t)|^{2} & +m_{\mathcal{A}}\|\dot{\boldsymbol{u}}(t)\|^{2}+\alpha\|\Theta \boldsymbol{u}(t)\|^{2} \\
\leq & |\ddot{\boldsymbol{u}}(0)|^{2}+a\left(\boldsymbol{v}_{0}, \boldsymbol{v}_{0}\right)+\left\langle\Theta^{*} B^{-1} \Theta \boldsymbol{v}_{0}, \boldsymbol{v}_{0}\right\rangle_{V^{\prime}, V}+2\langle\dot{\boldsymbol{h}}(t), \dot{\boldsymbol{u}}(t)\rangle_{V^{\prime}, V} \\
& -2\left\langle\dot{\boldsymbol{h}}(0), \boldsymbol{v}_{0}\right\rangle_{V^{\prime}, V}-2 \int_{0}^{t}\langle\ddot{\boldsymbol{h}}(s), \dot{\boldsymbol{u}}(s)\rangle_{V^{\prime}, V} d s .
\end{aligned}
$$

In order to estimate $|\ddot{\boldsymbol{u}}(0)|$, we put $t=0$ in (4.19) and use (4.20) to find $(\ddot{\boldsymbol{u}}(0), \boldsymbol{v})+a\left(\boldsymbol{u}_{0}, \boldsymbol{v}\right)+\left\langle\Theta^{*} B^{-1} \Theta \boldsymbol{u}_{0}, \boldsymbol{v}\right\rangle_{V^{\prime}, V}+\left\langle J_{\epsilon}\left(\boldsymbol{v}_{0}\right), \boldsymbol{v}\right\rangle_{V^{\prime}, V}=\langle\boldsymbol{h}(0), \boldsymbol{v}\rangle_{V^{\prime}, V}$, for all $\boldsymbol{v} \in V^{m}$. Keeping in mind (3.19) we have $\left\langle J_{\epsilon}\left(\boldsymbol{v}_{0}\right), \boldsymbol{v}\right\rangle_{V^{\prime}, V}=0$ and therefore

$$
(\ddot{\boldsymbol{u}}(0), \boldsymbol{v})=-a\left(\boldsymbol{u}_{0}, \boldsymbol{v}\right)-\left\langle\Theta^{*} B^{-1} \Theta \boldsymbol{u}_{0}, \boldsymbol{v}\right\rangle_{V^{\prime}, V}+\langle\boldsymbol{h}(0), \boldsymbol{v}\rangle_{V^{\prime}, V},
$$

for all $\boldsymbol{v} \in V^{m}$. Using the definition of $\boldsymbol{h}(0)$, (4.10) and (4.27) we get

$$
(\ddot{\boldsymbol{u}}(0), \boldsymbol{v})=\left(\operatorname{Div} \boldsymbol{\sigma}_{0}+\boldsymbol{f}_{0}(0), \boldsymbol{v}\right)_{H}, \forall \boldsymbol{v} \in V^{m}
$$

which implies

$$
\rho \ddot{\boldsymbol{u}}(0)=\operatorname{Div} \boldsymbol{\sigma}_{0}+\boldsymbol{f}_{0}(0) \text { a.e. in } \Omega \text {. }
$$

Hence, $|\ddot{\boldsymbol{u}}(0)|$ is bounded. Therefore, from equation (4.26) and assumptions in data, we can show that there exists $C>0$ such that

$$
|\ddot{\boldsymbol{u}}(t)|^{2}+\|\dot{\boldsymbol{u}}(t)\|^{2} \leq C+C \int_{0}^{t}\left(|\ddot{\boldsymbol{u}}(\theta)|^{2}+\|\dot{\boldsymbol{u}}(\theta)\|^{2}\right) d \theta
$$

Now, by using the Gronwall's inequality, it is straightforward to find the following estimates:

$$
\left\{\begin{array}{l}
\left\{\dot{\boldsymbol{u}}_{\epsilon}^{m}\right\}_{m, \epsilon} \text { is bounded in } L^{\infty}(0, T ; V) \\
\left\{\ddot{\boldsymbol{u}}_{\epsilon}^{m}\right\}_{m, \epsilon} \text { is bounded in } L^{\infty}(0, T ; H)
\end{array}\right.
$$

PAssage to The Limit IN $m$ AND $\epsilon$. The a priori estimates (4.23) and (4.29) are used now to deduce that there exists $\boldsymbol{u} \in W^{1, \infty}(0, T ; V) \cap W^{2, \infty}(0, T ; H)$, and a subsequence of $\left\{\boldsymbol{u}_{\epsilon}^{m}\right\}_{m, \epsilon}$ such that for $m \rightarrow \infty$ and $\epsilon \rightarrow 0$, we have:

$$
\begin{gathered}
\boldsymbol{u}_{\epsilon}^{m} \rightarrow \boldsymbol{u} \text { and } \dot{\boldsymbol{u}}_{\epsilon}^{m} \rightarrow \dot{\boldsymbol{u}} \text { weak } * \text { in } L^{\infty}(0, T ; V), \\
\ddot{\boldsymbol{u}}_{\epsilon}^{m} \rightarrow \ddot{\boldsymbol{u}} \text { weak } * \text { in } L^{\infty}(0, T ; H),
\end{gathered}
$$

Since $W^{1, \infty}(0, T ; V) \hookrightarrow C([0, T] ; V)$ we deduce that $\boldsymbol{u}_{\epsilon}^{m}(T) \rightarrow \boldsymbol{u}(T)$ weakly in $V$. Now, if we denote $Q_{T}=\Omega \times(0, T)$, we obtain from (4.23) and (4.29) that

$$
\left\{\boldsymbol{u}_{\epsilon}^{m}\right\}_{\epsilon, m} \text { and }\left\{\dot{\boldsymbol{u}}_{\epsilon}^{m}\right\}_{\epsilon, m} \text { are bounded in }\left[H^{1}\left(Q_{T}\right)\right]^{d} \text {. }
$$


Keeping in mind that $\partial Q_{T}=(\partial \Omega \times] 0, T[) \cup(\Omega \times\{\mathbf{0}\}) \cup(\Omega \times\{T\})$ and since the trace map from $H^{1}\left(Q_{T}\right)$ to $L^{2}\left(\partial Q_{T}\right)$ is completely continuous, we find

$$
\left\{\begin{array}{l}
\text { (a) } \boldsymbol{u}_{\epsilon}^{m} \rightarrow \boldsymbol{u}, \dot{\boldsymbol{u}}_{\epsilon}^{m} \rightarrow \dot{\boldsymbol{u}} \text { strongly in } L^{2}(\Gamma \times] 0, T[)^{d}, \\
\text { (b) } \boldsymbol{u}_{\epsilon}^{m}(T) \rightarrow \boldsymbol{u}(T), \dot{\boldsymbol{u}}_{\epsilon}^{m}(T) \rightarrow \dot{\boldsymbol{u}}(T) \text { strongly in } H \\
\text { (c) } \boldsymbol{u}_{\epsilon}^{m}(0) \rightarrow \boldsymbol{u}(0), \dot{\boldsymbol{u}}_{\epsilon}^{m}(0) \rightarrow \dot{\boldsymbol{u}}(0) \text { strongly in } H
\end{array}\right.
$$

We prove now that $\boldsymbol{u}$ is a solution of a variational problem (4.8)-(4.9). To this end, let $\boldsymbol{w} \in L^{2}(0, T ; V)$ and $\left\{\boldsymbol{w}^{m}\right\} \subset L^{2}\left(0, T ; V^{m}\right)$ such that $\boldsymbol{w}^{m} \rightarrow$ $\boldsymbol{w}$ strongly in $L^{2}(0, T ; V)$. Let us remark that the convexity of $j_{\epsilon}$ and the inequality (4.17) imply that

$$
\left\langle J_{\epsilon}(\boldsymbol{z}), \boldsymbol{w}-\boldsymbol{z}\right\rangle_{V^{\prime}, V} \leq j_{\epsilon}(\boldsymbol{w})-j_{\epsilon}(\boldsymbol{z}) \leq j(\boldsymbol{w})-j(\boldsymbol{z})+2 C \epsilon, \quad \forall \boldsymbol{z}, \boldsymbol{w} \in V
$$

If we use the inequality (4.31), we take $\boldsymbol{v}=\boldsymbol{w}^{m}(t)-\dot{\boldsymbol{u}}_{\epsilon}^{m}(t)$ in (4.19) and integrate (4.19) from 0 to $T$, we get

$$
\begin{aligned}
2 C T & +\int_{0}^{T}\left(\left(\ddot{\boldsymbol{u}}_{\epsilon}^{m}(t), \boldsymbol{w}^{m}(t)-\dot{\boldsymbol{u}}_{\epsilon}^{m}(t)\right)+a\left(\boldsymbol{u}_{\epsilon}^{m}(t), \boldsymbol{w}^{m}(t)-\dot{\boldsymbol{u}}_{\epsilon}^{m}(t)\right)\right) d t \\
& +\int_{0}^{T}\left\langle\Theta^{*} B^{-1} \Theta \boldsymbol{u}_{\epsilon}^{m}(t), \boldsymbol{w}^{m}(t)-\dot{\boldsymbol{u}}_{\epsilon}^{m}(t)\right\rangle_{V^{\prime}, V} d t \\
& +\int_{0}^{T}\left(j\left(\boldsymbol{w}^{m}(t)\right)-j\left(\dot{\boldsymbol{u}}_{\epsilon}^{m}(t)\right)\right) d t \\
\geq & \int_{0}^{T}\left\langle\boldsymbol{f}(t)-\Theta^{*} B^{-1} q(t), \boldsymbol{w}^{m}(t)-\dot{\boldsymbol{u}}_{\epsilon}^{m}(t)\right\rangle_{V^{\prime}, V} d t .
\end{aligned}
$$

The last inequality implies

$$
\begin{aligned}
2 \epsilon C T & +\int_{0}^{T}\left(\left(\ddot{\boldsymbol{u}}_{\epsilon}^{m}(t), \boldsymbol{w}^{m}(t)\right)+a\left(\boldsymbol{u}_{\epsilon}^{m}(t), \boldsymbol{w}^{m}(t)\right)\right. \\
& \left.+\left\langle\Theta^{*} B^{-1} \Theta \boldsymbol{u}_{\epsilon}^{m}(t), \boldsymbol{w}^{m}(t)\right\rangle_{V^{\prime}, V}\right) d t \\
& +\frac{1}{2}\left(\left|\boldsymbol{v}_{0}\right|^{2}-\left|\dot{\boldsymbol{u}}_{\epsilon}^{m}(T)\right|^{2}+a\left(\boldsymbol{u}_{0}, \boldsymbol{u}_{0}\right)+\left\langle\Theta^{*} B^{-1} \Theta \boldsymbol{u}_{0}, \boldsymbol{u}_{0}\right\rangle_{V^{\prime}, V}\right) \\
& +\int_{0}^{T}\left(j\left(\boldsymbol{w}^{m}(t)\right)-j\left(\dot{\boldsymbol{u}}_{\epsilon}^{m}(t)\right)\right) d t-\int_{0}^{T}\left\langle\boldsymbol{h}(t), \boldsymbol{w}^{m}(t)-\dot{\boldsymbol{u}}_{\epsilon}^{m}(t)\right\rangle_{V^{\prime}, V} d t \\
\geq & \frac{1}{2}\left(a\left(\boldsymbol{u}_{\epsilon}^{m}(T), \boldsymbol{u}_{\epsilon}^{m}(T)\right)+\left\langle\Theta^{*} B^{-1} \Theta \boldsymbol{u}_{\epsilon}^{m}(T), \boldsymbol{u}_{\epsilon}^{m}(T)\right\rangle_{V^{\prime}, V}\right) .
\end{aligned}
$$

Since $\boldsymbol{w}^{m} \rightarrow \boldsymbol{w}$ strongly in $L^{2}(0, T ; V)$ then having in mind (4.30) we find

$$
\int_{0}^{T}\left(j\left(\boldsymbol{w}^{m}(t)\right)-j\left(\dot{\boldsymbol{u}}_{\epsilon}^{m}(t)\right)\right) d t \rightarrow \int_{0}^{T}(j(\boldsymbol{w}(t))-j(\dot{\boldsymbol{u}}(t))) d t .
$$


Thus the left member of the inequality (4.32) has a limit when $\epsilon \rightarrow 0$ and $m \rightarrow \infty$, if we pass to lower limit in (4.32) with $\epsilon \rightarrow 0$ and $m \rightarrow \infty$ we have

$$
\begin{aligned}
\int_{0}^{T} & \left((\ddot{\boldsymbol{u}}(t), \boldsymbol{w}(t))+a(\boldsymbol{u}(t), \boldsymbol{w}(t))+\left\langle\Theta^{*} B^{-1} \Theta \boldsymbol{u}, \boldsymbol{w}(t)\right\rangle_{V^{\prime}, V}\right) d t \\
(4.33)+ & +\frac{1}{2}\left(\left|\boldsymbol{v}_{0}\right|^{2}-|\dot{\boldsymbol{u}}(T)|^{2}+a\left(\boldsymbol{u}_{0}, \boldsymbol{u}_{0}\right)+\left\langle\Theta^{*} B^{-1} \Theta \boldsymbol{u}_{0}, \boldsymbol{u}_{0}\right\rangle_{V^{\prime}, V}\right) \\
& +\int_{0}^{T}(j(\boldsymbol{w}(t))-j(\dot{\boldsymbol{u}}(t))) d t-\int_{0}^{T}\langle\boldsymbol{h}(t), \boldsymbol{w}(t)-\dot{\boldsymbol{u}}(t)\rangle_{V^{\prime}, V} d t \\
\geq & \frac{1}{2} \lim _{m \rightarrow+\infty, \epsilon \rightarrow 0} \inf \left(a\left(\boldsymbol{u}_{\epsilon}^{m}(T), \boldsymbol{u}_{\epsilon}^{m}(T)\right)+\left\langle\Theta^{*} B^{-1} \Theta \boldsymbol{u}_{\epsilon}^{m}(T), \boldsymbol{u}_{\epsilon}^{m}(T)\right\rangle_{V^{\prime}, V}\right) \\
\geq & \frac{1}{2}\left(a(\boldsymbol{u}(T), \boldsymbol{u}(T))+\left\langle\Theta^{*} B^{-1} \Theta \boldsymbol{u}(T), \boldsymbol{u}(T)\right\rangle_{V^{\prime}, V}\right) .
\end{aligned}
$$

From this inequality, we find

$$
\begin{aligned}
& \int_{0}^{T}\left((\ddot{\boldsymbol{u}}(t), \boldsymbol{w}(t)-\dot{\boldsymbol{u}}(t))+a(\boldsymbol{u}(t), \boldsymbol{w}(t)-\dot{\boldsymbol{u}}(t))+\left\langle\Theta^{*} B^{-1} \Theta \boldsymbol{u}(t), \boldsymbol{w}(t)-\dot{\boldsymbol{u}}(t)\right\rangle_{V^{\prime}, V}\right) d t \\
& +\int_{0}^{T}(j(\boldsymbol{w}(t))-j(\dot{\boldsymbol{u}}(t))) d t \geq \int_{0}^{T}\left\langle\boldsymbol{f}(t)-\Theta^{*} B^{-1} q(t), \boldsymbol{w}(t)-\dot{\boldsymbol{u}}(t)\right\rangle_{V^{\prime}, V} d t .
\end{aligned}
$$

From the last inequality and uniqueness result we deduce that $\boldsymbol{u}$ is the unique solution of the variational problem (4.8)-(4.9).

Finally, we define $\varphi$ by (4.10) and taking in mind Lemma 4.1, it is now easy to show that $(\boldsymbol{u}, \varphi)$ is the unique solution of Problem $P_{V}$.

\section{ASYMPTOTIC BEHAVIOR FOR VANISHING VISCOSITY}

In this section we consider a viscous perturbation of problem $P$. To this end let $\eta>0$ be a small viscosity parameter and let us replace the piezoelectric law (2.3)-(2.4) in $P$ with the following linear piezo-viscoelastic constitutive equations (see [7]), (for the case where the constitutive equation is viscoelastic without piezoelectricity effect see $([3,11]))$ :

$$
\begin{gathered}
\boldsymbol{\sigma}_{\eta}=\mathcal{A} \boldsymbol{\varepsilon}\left(\boldsymbol{u}_{\eta}\right)+\eta \mathcal{C} \varepsilon\left(\dot{\boldsymbol{u}}_{\eta}\right)-\mathcal{E}^{*} \boldsymbol{E}\left(\varphi_{\eta}\right) \quad \text { in } \Omega \times(0, T), \\
\boldsymbol{D}_{\eta}=\mathcal{E} \boldsymbol{\varepsilon}\left(\boldsymbol{u}_{\eta}\right)+\boldsymbol{\beta} \boldsymbol{E}\left(\varphi_{\eta}\right) \quad \text { in } \Omega \times(0, T),
\end{gathered}
$$

where $\mathcal{C}=\left(c_{i j k l}\right)$ is a (fourth-order) viscosity tensor which is bounded, symmetric and positively defined, i.e.

$$
c_{i j k l} \in L^{\infty}(\Omega), \quad \mathcal{C}(\boldsymbol{x}) \boldsymbol{\varepsilon} \cdot \boldsymbol{\sigma}=\mathcal{C}(\boldsymbol{x}) \boldsymbol{\sigma} \cdot \boldsymbol{\varepsilon}, \quad \mathcal{C}(\boldsymbol{x}) \boldsymbol{\varepsilon} \cdot \boldsymbol{\varepsilon} \geq c_{0}|\boldsymbol{\varepsilon}|^{2}
$$

a.e $\boldsymbol{x} \in \Omega, \forall i, j, k, l=1, \ldots, d$ and for all $\boldsymbol{\sigma}, \boldsymbol{\varepsilon} \in \mathbb{S}^{d}$.

The viscous piezoelectric problem $P^{\eta}$ is as follows

Problem $P^{\eta}$. Find a displacement field $\boldsymbol{u}_{\eta}: \Omega \times[0, T] \rightarrow \mathbb{R}^{d}$, a stress field $\boldsymbol{\sigma}_{\eta}: \Omega \times[0, T] \rightarrow \mathbb{S}^{d}$, an electric potential field $\varphi_{\eta}: \Omega \times[0, T] \rightarrow \mathbb{R}$ and 
an electric displacement field $\boldsymbol{D}_{\eta}: \Omega \times[0, T] \rightarrow \mathbb{R}^{d}$ such that (5.1)-(5.2),(2.1)(2.2) and (2.5)-(2.10) hold.

Now, if we denote by $c: V \times V \rightarrow \mathbb{R}$ the following bilinear, symmetric and coercive application:

$$
c(\boldsymbol{u}, \boldsymbol{v}):=\int_{\Omega} \mathcal{C} \varepsilon(\boldsymbol{u}) \cdot \varepsilon(\boldsymbol{v}) d \boldsymbol{x}:=(\mathcal{C} \varepsilon(\boldsymbol{u}), \varepsilon(\boldsymbol{v}))_{Q},
$$

then the variational formulation of $P^{\eta}$ is:

Problem $P_{V}^{\eta}$. Find the displacement field $\boldsymbol{u}_{\eta}:[0, T] \rightarrow V$ and the electric potential $\varphi_{\eta}:[0, T] \rightarrow W$ such that $\forall \boldsymbol{v} \in V, \forall \psi \in W, \forall t \in[0, T]$

$$
\begin{gathered}
\left(\ddot{\boldsymbol{u}}_{\eta}(t), \boldsymbol{v}-\dot{\boldsymbol{u}}_{\eta}(t)\right)+a\left(\boldsymbol{u}_{\eta}(t), \boldsymbol{v}-\dot{\boldsymbol{u}}_{\eta}(t)\right)+\eta c\left(\dot{\boldsymbol{u}}_{\eta}(t), \boldsymbol{v}-\dot{\boldsymbol{u}}_{\eta}(t)\right) \\
+e\left(\boldsymbol{v}-\dot{\boldsymbol{u}}_{\eta}(t), \varphi_{\eta}(t)\right)+j(\boldsymbol{v})-j\left(\dot{\boldsymbol{u}}_{\eta}(t)\right) \geq\left\langle\boldsymbol{f}(t), \boldsymbol{v}-\dot{\boldsymbol{u}}_{\eta}(t)\right\rangle_{V^{\prime}, V} \\
\left.b\left(\varphi_{\eta}(t), \psi\right)-e\left(\boldsymbol{u}_{\eta}(t)\right), \psi\right)=\langle q(t), \psi\rangle_{W^{\prime}, W} \\
\boldsymbol{u}_{\eta}(0)=\boldsymbol{u}_{0}, \quad \dot{\boldsymbol{u}}_{\eta}(0)=\boldsymbol{v}_{0} .
\end{gathered}
$$

We shall add the following condition:

$$
\text { there exists } \boldsymbol{v}_{1} \in H \text { such that } c\left(\boldsymbol{v}_{0}, v\right)=\left(\boldsymbol{v}_{1}, v\right) \forall \boldsymbol{v} \in V .
$$

We have the following existence and uniqueness result:

Theorem 5.1. Assume that (5.1)-(5.3), (3.2)-(3.4), (3.9)-(3.14) and (3.18)-(3.19) hold. Then, for all $\eta>0$, there exists a unique solution $\left(\boldsymbol{u}_{\eta}, \varphi_{\eta}\right)$ of Problem $P_{V}^{\eta}$ such that

$$
\boldsymbol{u}_{\eta} \in W^{1, \infty}(0, T ; V) \cap W^{2, \infty}(0, T ; H) \text { and } \varphi_{\eta} \in W^{1, \infty}(0, T ; W) .
$$

In addition, if we assume that (5.8) hold. Then there exists a subsequence of $\left\{\boldsymbol{u}_{\eta}, \varphi_{\eta}\right\}_{\eta}$, denoted again by $\left\{\boldsymbol{u}_{\eta}, \varphi_{\eta}\right\}_{\eta}$, such that when $\eta \rightarrow 0$ we have

$$
\begin{aligned}
& \boldsymbol{u}_{\eta} \rightarrow \boldsymbol{u} \text { and } \dot{\boldsymbol{u}}_{\eta} \rightarrow \dot{\boldsymbol{u}} \text { weak }{ }^{*} \text { in } L^{\infty}(0, T ; V) \text {, } \\
& \ddot{\boldsymbol{u}}_{\eta} \rightarrow \ddot{\boldsymbol{u}} \text { weak }^{*} \text { in } L^{\infty}(0, T ; H) \text {, } \\
& \varphi_{\eta} \rightarrow \varphi \text { and } \dot{\varphi}_{\eta} \rightarrow \dot{\varphi} \text { weak }{ }^{*} \text { in } L^{\infty}(0, T ; W)
\end{aligned}
$$

where the couple $(\boldsymbol{u}, \varphi)$ is the unique solution of $P_{V} .$.

Proof. The proof of Theorem 5.1 will be carried out in several steps.

UNiquenEss. For all $\eta>0$ we have $\eta c(\dot{\boldsymbol{u}}, \dot{\boldsymbol{u}}) \geq 0$ then the uniqueness result of Theorem 5.1 is proved in the same way that in Section 4.

Existence. In order to prove the existence result, we start with the following equivalence result. 
LEMMA 5.2. Let $\eta>0$. The couple $\left(\boldsymbol{u}_{\eta}, \varphi_{\eta}\right)$ is a unique solution to problem $P_{V}^{\eta}$ if only if for all $\boldsymbol{v}$ in $V$ we have:

$$
\begin{gathered}
\left(\ddot{\boldsymbol{u}}_{\eta}(t), \boldsymbol{v}-\dot{\boldsymbol{u}}_{\eta}(t)\right)+a\left(\boldsymbol{u}_{\eta}(t), \boldsymbol{v}-\dot{\boldsymbol{u}}_{\eta}(t)\right)+\eta c\left(\boldsymbol{u}_{\eta}(t), \boldsymbol{v}-\dot{\boldsymbol{u}}_{\eta}(t)\right) \\
+\left\langle\Theta^{*} B^{-1} \Theta \boldsymbol{u}_{\eta}(t), \boldsymbol{v}-\dot{\boldsymbol{u}}_{\eta}(t)\right\rangle_{V^{\prime}, V}+j(\boldsymbol{v})-j\left(\dot{\boldsymbol{u}}_{\eta}(t)\right) \\
\geq\left\langle\boldsymbol{f}(t)-\Theta^{*} B^{-1} q(t), \boldsymbol{v}-\dot{\boldsymbol{u}}_{\eta}(t)\right\rangle_{V^{\prime}, V}, \\
\varphi_{\eta}(t)=B^{-1} \Theta \boldsymbol{u}_{\eta}(t)+B^{-1} q(t), \forall t \in[0, T] .
\end{gathered}
$$

with the initials conditions (5.7).

Let us observe that this lemma can be proved in the same way that Lemma 4.1 .

Now, as in Section 4, we consider the regularized problem of (5.10) with the initials conditions (5.7): find $\boldsymbol{u}_{\epsilon \eta}:[0, T] \rightarrow V$ such that

$$
\begin{gathered}
\left(\ddot{\boldsymbol{u}}_{\epsilon \eta}(t), \boldsymbol{v}\right)+a\left(\boldsymbol{u}_{\epsilon \eta}(t), \boldsymbol{v}\right)+\eta c\left(\dot{\boldsymbol{u}}_{\epsilon \eta}(t), \boldsymbol{v}\right)+\left\langle\Theta^{*} B^{-1} \Theta \boldsymbol{u}_{\epsilon \eta}(t), \boldsymbol{v}\right\rangle_{V^{\prime}, V} \\
+\left\langle J_{\epsilon}\left(\dot{\boldsymbol{u}}_{\epsilon \eta}(t), \boldsymbol{v}\right\rangle_{V^{\prime}, V}\right. \\
=\langle\boldsymbol{f}(t), \boldsymbol{v}\rangle_{V^{\prime}, V}-\left\langle\Theta^{*} B^{-1} q(t), \boldsymbol{v}\right\rangle_{V^{\prime}, V}, \forall \boldsymbol{v} \in V, \forall t \in[0, T] \\
\boldsymbol{u}_{\epsilon \eta}(0)=\boldsymbol{u}_{0}, \quad \dot{\boldsymbol{u}}_{\epsilon \eta}(0)=\boldsymbol{v}_{0} .
\end{gathered}
$$

Estimate I. Since $\left\langle J_{\epsilon}(\boldsymbol{v}), \boldsymbol{v}\right\rangle_{V^{\prime}, V} \geq 0$ for all $\boldsymbol{v} \in V$, if we take $\boldsymbol{v}=\dot{\boldsymbol{u}}_{\epsilon \eta}$ in $(5.12)$, we obtain

$$
\begin{aligned}
& \frac{1}{2} \frac{d}{d t}\left(\left|\dot{\boldsymbol{u}}_{\epsilon \eta}(t)\right|^{2}+a\left(\boldsymbol{u}_{\epsilon \eta}(t), \boldsymbol{u}_{\epsilon \eta}(t)\right)+\left\langle\Theta^{*} B^{-1} \Theta \boldsymbol{u}_{\epsilon \eta}, \boldsymbol{u}_{\epsilon \eta}\right\rangle_{V^{\prime}, V}\right) \\
& +\eta c\left(\dot{\boldsymbol{u}}_{\epsilon \eta}(t), \dot{\boldsymbol{u}}_{\epsilon \eta}(t)\right) \leq\left\langle\boldsymbol{h}(t), \dot{\boldsymbol{u}}_{\epsilon \eta}(t)\right\rangle_{V^{\prime}, V}
\end{aligned}
$$

where $\boldsymbol{h}(t)=\boldsymbol{f}(t)-\Theta^{*} B^{-1} q(t)$. Using the same techniques of Section 4 , we can easily deduce:

$$
\left\|\boldsymbol{u}_{\epsilon \eta}(t)\right\|+\left|\dot{\boldsymbol{u}}_{\epsilon \eta}(t)\right| \leq C, \quad \eta \int_{0}^{T}\left\|\dot{\boldsymbol{u}}_{\epsilon \eta}(t)\right\|^{2} d t \leq C .
$$

Estimate II. Setting $\boldsymbol{v}=\ddot{\boldsymbol{u}}_{\epsilon \eta}(t)$ in (5.12) we obtain

$$
\begin{aligned}
& \left|\ddot{\boldsymbol{u}}_{\epsilon \eta}(t)\right|^{2}+a\left(\boldsymbol{u}_{\epsilon \eta}(t), \ddot{\boldsymbol{u}}_{\epsilon \eta}(t)\right)+\left\langle\Theta^{*} B^{-1} \Theta \boldsymbol{u}_{\epsilon \eta}(t), \ddot{\boldsymbol{u}}_{\epsilon \eta}(t)\right\rangle_{V^{\prime}, V} \\
& \quad+\frac{\eta}{2} \frac{d}{d t} c\left(\dot{\boldsymbol{u}}_{\epsilon \eta}(t), \dot{\boldsymbol{u}}_{\epsilon \eta}(t)\right)+\left\langle J_{\epsilon}\left(\dot{\boldsymbol{u}}_{\epsilon \eta}(t)\right), \ddot{\boldsymbol{u}}_{\epsilon \eta}(t)\right\rangle_{V^{\prime}, V} \\
& =\left\langle\boldsymbol{h}(t), \ddot{\boldsymbol{u}}_{\epsilon \eta}(t)\right\rangle_{V^{\prime}, V} .
\end{aligned}
$$

We have

$$
\begin{aligned}
\left\langle J_{\epsilon}(\dot{\boldsymbol{u}}(t)), \ddot{\boldsymbol{u}}(t)\right\rangle_{V^{\prime}, V} & =\int_{\Gamma_{3}} S(s) \mu(s) \Psi_{\epsilon}^{\prime}\left(\dot{\boldsymbol{u}}_{\epsilon \eta \tau}(t)\right)\left(\ddot{\boldsymbol{u}}_{\epsilon \eta \tau}(t)\right) d s \\
& =\int_{\Gamma_{3}} S(s) \mu(s) \frac{\partial}{\partial t} \Psi_{\epsilon}\left(\dot{\boldsymbol{u}}_{\epsilon \eta \tau}(t)\right) d s .
\end{aligned}
$$


Now, using coercivity of the form $c(\cdot, \cdot)$, the last equality and integrating over $(0, t), 0 \leq t \leq T$, the equation (5.16) we get

$$
\begin{aligned}
& \int_{0}^{t}\left|\ddot{\boldsymbol{u}}_{\epsilon \eta}(\theta)\right|^{2} d \theta+C \frac{\eta}{2}\left\|\dot{\boldsymbol{u}}_{\epsilon \eta}(t)\right\|^{2} \leq \frac{\eta}{2} c\left(\boldsymbol{v}_{0}, \boldsymbol{v}_{0}\right) \\
& \quad-\int_{0}^{t} a\left(\boldsymbol{u}_{\epsilon \eta}(\theta), \ddot{\boldsymbol{u}}_{\epsilon \eta}(\theta)\right) d \theta-\int_{0}^{t}\left\langle\Theta^{*} B^{-1} \Theta \boldsymbol{u}_{\epsilon \eta}(\theta), \ddot{\boldsymbol{u}}_{\epsilon \eta}(\theta)\right\rangle_{V^{\prime}, V} d \theta \\
& +\int_{0}^{t}\left\langle\boldsymbol{h}(\theta), \ddot{\boldsymbol{u}}_{\epsilon \eta}(\theta)\right\rangle_{V^{\prime}, V} d \theta-\int_{0}^{t} \int_{\Gamma_{3}} S(s) \mu(s) \frac{\partial}{\partial \theta} \Psi_{\epsilon}\left(\dot{\boldsymbol{u}}_{\epsilon \eta \tau}(s, \theta)\right) d s d \theta
\end{aligned}
$$

After integration by parts, we obtain

$$
\begin{aligned}
& \int_{0}^{t}\left|\ddot{\boldsymbol{u}}_{\epsilon \eta}(\theta)\right|^{2} d \theta+\frac{\eta}{2}\left\|\dot{\boldsymbol{u}}_{\epsilon \eta}(t)\right\|^{2} \leq C-a\left(\boldsymbol{u}_{\epsilon \eta}(t), \dot{\boldsymbol{u}}_{\epsilon \eta}(t)\right) \\
& (5.18)-\left\langle\Theta^{*} B^{-1} \Theta \boldsymbol{u}_{\epsilon \eta}(t), \dot{\boldsymbol{u}}_{\epsilon \eta}(t)\right\rangle_{V^{\prime}, V}+\left\langle\boldsymbol{h}(t), \dot{\boldsymbol{u}}_{\epsilon \eta}(t)\right\rangle_{V^{\prime}, V} \\
& +\int_{0}^{t} a\left(\dot{\boldsymbol{u}}_{\epsilon \eta}(\theta), \dot{\boldsymbol{u}}_{\epsilon \eta}(\theta)\right) d \theta+\int_{0}^{t}\left\langle\Theta^{*} B^{-1} \Theta \dot{\boldsymbol{u}}_{\epsilon \eta}(\theta), \dot{\boldsymbol{u}}_{\epsilon \eta}(\theta)\right\rangle_{V^{\prime}, V} d \theta \\
& -\int_{0}^{t}\left\langle\dot{\boldsymbol{h}}(\theta), \dot{\boldsymbol{u}}_{\epsilon \eta}(\theta)\right\rangle_{V^{\prime}, V} d \theta-\int_{0}^{t} \int_{\Gamma_{3}} S(s) \mu(s) \frac{\partial}{\partial \theta} \Psi_{\epsilon}\left(\dot{\boldsymbol{u}}_{\epsilon \eta \tau}(s, \theta)\right) d s d \theta .
\end{aligned}
$$

Keeping in mind (3.19), (3.9), (3.11), (4.12) and trace theorem, we have

$$
\begin{aligned}
& \left|\int_{0}^{t} \int_{\Gamma_{3}} S(s) \mu(s) \frac{\partial}{\partial \theta} \Psi_{\epsilon}\left(\dot{\boldsymbol{u}}_{\epsilon \eta \tau}(s, \theta)\right) d s d \theta\right| \\
& \quad=\mid \int_{\Gamma_{3}} S(s) \mu(s) \Psi_{\epsilon}\left(\left.\dot{\boldsymbol{u}}_{\epsilon \eta \tau}(t) d s|\leq C| \dot{\boldsymbol{u}}_{\epsilon \eta \tau}(t)\right|_{L^{2}\left(\Gamma_{3}\right)} \leq C\left\|\dot{\boldsymbol{u}}_{\epsilon \eta}(t)\right\| .\right.
\end{aligned}
$$

Therefore, using inequalities (5.15) and (5.18), some easy Young inequalities and assumptions on data, we get

$$
\int_{0}^{t}\left|\ddot{\boldsymbol{u}}_{\epsilon \eta}(\theta)\right|^{2} d \theta+\frac{\eta}{4}\left\|\dot{\boldsymbol{u}}_{\epsilon \eta}(t)\right\|^{2} \leq C+C \int_{0}^{t}\left\|\dot{\boldsymbol{u}}_{\epsilon \eta}(\theta)\right\|^{2} d \theta
$$

where the generic constants $C$ are depending on data and $\eta$. Using the Gronwall's inequality, it is straightforward to find the following estimate: there exists $C(\eta)>0$ such that

$$
\eta\left\|\dot{\boldsymbol{u}}_{\epsilon \eta}(t)\right\|^{2}+\int_{0}^{T}\left|\ddot{\boldsymbol{u}}_{\epsilon \eta}(t)\right|^{2} d t \leq C(\eta)
$$

Now, using estimates (5.15) and (5.20) we prove, as in Section 4 by passing to limit in $\epsilon$, that for all fixed $\eta>0$, there exists a unique solution $\left(\boldsymbol{u}_{\eta}, \varphi_{\eta}\right)$ of Problem $P_{V}^{\eta}$ such that

$$
\boldsymbol{u}_{\eta} \in W^{1, \infty}(0, T ; V) \cap W^{2,2}(0, T ; H) \text { and } \varphi_{\eta} \in W^{1, \infty}(0, T ; W) .
$$


Convergence. We note that all constants considered in the following are independent of the viscosity parameter $\eta$. Now, differentiating the equation (5.12) with respect to $t$ and replacing $\boldsymbol{v}$ by $\ddot{\boldsymbol{u}}_{\epsilon \eta}(t)$ we have, as in Section 4

$$
\begin{aligned}
& \frac{1}{2} \frac{d}{d t}\left(\left|\ddot{\boldsymbol{u}}_{\epsilon \eta}(t)\right|^{2}+a\left(\dot{\boldsymbol{u}}_{\epsilon \eta}(t), \dot{\boldsymbol{u}}_{\epsilon \eta}(t)+\left\langle\Theta^{*} B^{-1} \Theta \dot{\boldsymbol{u}}_{\epsilon \eta}(t), \dot{\boldsymbol{u}}_{\epsilon \eta}(t)\right\rangle_{V^{\prime}, V}\right)\right. \\
& \left.\quad+\eta c\left(\ddot{\boldsymbol{u}}_{\epsilon \eta}(t), \ddot{\boldsymbol{u}}_{\epsilon \eta}(t)\right)\right) \leq\left\langle\dot{\boldsymbol{h}}(t), \ddot{\boldsymbol{u}}_{\epsilon \eta}(t)\right\rangle_{V^{\prime}, V} .
\end{aligned}
$$

Now, using coercivity of the form $a(\cdot, \cdot)$ together with (4.24) and integrating over $(0, t), 0 \leq t \leq T$, we get

$$
\begin{gathered}
\left|\ddot{\boldsymbol{u}}_{\epsilon \eta}(t)\right|^{2}+m_{\mathcal{A}}\left\|\dot{\boldsymbol{u}}_{\epsilon \eta}(t)\right\|^{2}+\alpha\left\|\Theta \dot{\boldsymbol{u}}_{\epsilon \eta}(t)\right\|^{2}+C \eta \int_{0}^{t}\left\|\ddot{\boldsymbol{u}}_{\epsilon \eta}(\theta)\right\|^{2} d \theta \\
(5.22) \leq\left|\ddot{\boldsymbol{u}}_{\epsilon \eta}(0)\right|^{2}+a\left(\boldsymbol{v}_{0}, \boldsymbol{v}_{0}\right)+\left\langle\Theta^{*} B^{-1} \Theta \boldsymbol{v}_{0}, \boldsymbol{v}_{0}\right\rangle_{V^{\prime}, V}+2\left\langle\dot{\boldsymbol{h}}(t), \dot{\boldsymbol{u}}_{\epsilon \eta}(t)\right\rangle_{V^{\prime}, V} \\
-2\left\langle\dot{\boldsymbol{h}}(0), \boldsymbol{v}_{0}\right\rangle_{V^{\prime}, V}-2 \int_{0}^{t}\left\langle\ddot{\boldsymbol{h}}(\theta), \dot{\boldsymbol{u}}_{\epsilon \eta}(\theta)\right\rangle_{V^{\prime}, V} d \theta
\end{gathered}
$$

In order to estimate $\left|\ddot{\boldsymbol{u}}_{\epsilon \eta}(0)\right|$, we put $t=0$ in (5.12) and use (5.13), (5.8) to find

$$
\rho \ddot{\boldsymbol{u}}_{\epsilon \eta}(0)=\operatorname{Div} \boldsymbol{\sigma}_{0}+\boldsymbol{f}_{0}(0)-\eta \boldsymbol{v}_{1} \text { a.e. in } \Omega .
$$

Hence, $\left|\ddot{\boldsymbol{u}}_{\epsilon \eta}(0)\right|$ is bounded. Therefore, from equation (5.22) and assumptions in data, and by using the Gronwall's inequality, it is straightforward to find the following estimates: there exists $C>0$ such that

$$
\left\|\dot{\boldsymbol{u}}_{\epsilon \eta}(t)\right\|+\left|\ddot{\boldsymbol{u}}_{\epsilon \eta}(t)\right| \leq C, \quad \eta \int_{0}^{T}\left\|\ddot{\boldsymbol{u}}_{\epsilon \eta}(t)\right\|^{2} d t \leq C .
$$

From estimates (5.15) and (5.23), we have

$$
\left\|\boldsymbol{u}_{\epsilon \eta}(t)\right\|+\left\|\dot{\boldsymbol{u}}_{\epsilon \eta}(t)\right\|+\left|\ddot{\boldsymbol{u}}_{\epsilon \eta}(t)\right| \leq C, \quad \eta \int_{0}^{T}\left\|\ddot{\boldsymbol{u}}_{\epsilon \eta}(t)\right\|^{2} d t \leq C .
$$

Now, if we pass to limit in (5.24) with $\epsilon \rightarrow 0$ and by using the same techniques of convergence meted in Section 4 we find

$$
\boldsymbol{u}_{\eta} \in W^{1, \infty}(0, T ; V) \cap W^{2, \infty}(0, T ; H) \cap W^{2,2}(0, T ; V)
$$

which is the unique solution of (5.12)-(5.13) such that

$$
\left\|\boldsymbol{u}_{\eta}(t)\right\|+\left\|\dot{\boldsymbol{u}}_{\eta}(t)\right\|+\left|\ddot{\boldsymbol{u}}_{\eta}(t)\right| \leq C, \quad \eta \int_{0}^{T}\left\|\ddot{\boldsymbol{u}}_{\eta}(t)\right\|^{2} d t \leq C .
$$

From (5.26), we deduce that there exists a subsequence of $\left\{\boldsymbol{u}_{\eta}\right\}_{\eta}$ (again denoted by $\left\{\boldsymbol{u}_{\eta}\right\}_{\eta}$ ) such that when $\eta \rightarrow 0$ we have

$$
\begin{aligned}
& \boldsymbol{u}_{\eta} \rightarrow \boldsymbol{u} \text { and } \dot{\boldsymbol{u}}_{\eta} \rightarrow \dot{\boldsymbol{u}} \text { weak } * \text { in } L^{\infty}(0, T ; V), \\
& \ddot{\boldsymbol{u}}_{\eta} \rightarrow \ddot{\boldsymbol{u}} \text { weak } * \text { in } L^{\infty}(0, T ; H),
\end{aligned}
$$

where

$$
\boldsymbol{u} \in W^{1, \infty}(0, T ; V) \cap W^{2, \infty}(0, T ; H) .
$$


Let $\boldsymbol{v} \in L^{2}(0, T ; V)$ and pose $\boldsymbol{v}=\boldsymbol{v}(t)$, then by integrating (5.10) from 0 to $T$, we get

$$
\begin{aligned}
\int_{0}^{T}\left(\left(\ddot{\boldsymbol{u}}_{\eta}(t), \boldsymbol{v}(t)\right)+a\left(\boldsymbol{u}_{\eta}(t), \boldsymbol{v}(t)\right)+\left\langle\Theta^{*} B^{-1} \Theta \boldsymbol{u}_{\eta}(t), \boldsymbol{v}(t)\right\rangle_{V^{\prime}, V}\right) d t \\
(5.29)-\int_{0}^{T}\left(\left\langle\boldsymbol{h}(t), \boldsymbol{v}(t)-\dot{\boldsymbol{u}}_{\eta}(t)\right\rangle_{V^{\prime}, V}+\eta c\left(\dot{\boldsymbol{u}}_{\eta}(t), \boldsymbol{v}(t)\right)+j(\boldsymbol{v}(t))-j\left(\dot{\boldsymbol{u}}_{\eta}(t)\right)\right) d t \\
\geq \int_{0}^{T}\left(\left(\ddot{\boldsymbol{u}}_{\eta}(t), \dot{\boldsymbol{u}}_{\eta}(t)\right)+a\left(\boldsymbol{u}_{\eta}(t), \dot{\boldsymbol{u}}_{\eta}(t)\right)+\left\langle\Theta^{*} B^{-1} \Theta \boldsymbol{u}_{\eta}(t), \dot{\boldsymbol{u}}_{\eta}(t)\right\rangle_{V^{\prime}, V}\right) d t \\
=\frac{1}{2}\left(\left|\dot{\boldsymbol{u}}_{\eta}(T)\right|^{2}-\left|\boldsymbol{v}_{0}\right|^{2}+a\left(\boldsymbol{u}_{\eta}(T), \boldsymbol{u}_{\eta}(T)\right)-a\left(\boldsymbol{u}_{0}, \boldsymbol{u}_{0}\right)\right. \\
\left.\quad+\left\langle\Theta^{*} B^{-1} \Theta \boldsymbol{u}_{\eta}(T), \boldsymbol{u}_{\eta}(T)\right\rangle_{V^{\prime}, V}-\left\langle\Theta^{*} B^{-1} \Theta \boldsymbol{u}_{0}, \boldsymbol{u}_{0}\right\rangle_{V^{\prime}, V}\right)
\end{aligned}
$$

Using $\dot{\boldsymbol{u}}_{\eta}(T) \rightarrow \dot{\boldsymbol{u}}(T)$ strongly in $H, \boldsymbol{u}_{\eta}(T) \rightarrow \boldsymbol{u}(T)$ weakly in $V$ and the convexity of the continued forms on $V$ defined by: $\boldsymbol{v} \mapsto a(\boldsymbol{v}, \boldsymbol{v}), \boldsymbol{v} \mapsto$ $\left\langle\Theta^{*} B^{-1} \Theta \boldsymbol{v}, \boldsymbol{v}\right\rangle_{V^{\prime}, V}$, we have

$$
\begin{aligned}
\lim _{\eta \rightarrow 0} \inf & \left(\left|\dot{\boldsymbol{u}}_{\eta}(T)\right|^{2}-\left|\boldsymbol{v}_{0}\right|^{2}+a\left(\boldsymbol{u}_{\eta}(T), \boldsymbol{u}_{\eta}(T)\right)-a\left(\boldsymbol{u}_{0}, \boldsymbol{u}_{0}\right)\right. \\
& \left.+\left\langle\Theta^{*} B^{-1} \Theta \boldsymbol{u}_{\eta}(T), \boldsymbol{u}_{\eta}(T)\right\rangle_{V^{\prime}, V}-\left\langle\Theta^{*} B^{-1} \Theta \boldsymbol{u}_{0}, \boldsymbol{u}_{0}\right\rangle_{V^{\prime}, V}\right) \\
\geq & \left(|\dot{\boldsymbol{u}}(T)|^{2}-\left|\boldsymbol{v}_{0}\right|^{2}+a(\boldsymbol{u}(T), \boldsymbol{u}(T))-a\left(\boldsymbol{u}_{0}, \boldsymbol{u}_{0}\right)\right. \\
& \left.+\left\langle\Theta^{*} B^{-1} \Theta \boldsymbol{u}(T), \boldsymbol{u}(T)\right\rangle_{V^{\prime}, V}-\left\langle\Theta^{*} B^{-1} \Theta \boldsymbol{u}_{0}, \boldsymbol{u}_{0}\right\rangle_{V^{\prime}, V}\right) .
\end{aligned}
$$

Furthermore, from (5.26), (5.27) we obtain

$$
\begin{gathered}
\left|\int_{0}^{T} \eta c\left(\dot{\boldsymbol{u}}_{\eta}(t), \boldsymbol{v}(t)\right) d t\right| \leq C \eta\left\|\dot{\boldsymbol{u}}_{\eta}\right\|_{L^{\infty}(0, T ; V)}\|\boldsymbol{v}\|_{L^{2}(0, T ; V)} \leq C \eta \\
\int_{0}^{T} j\left(\dot{\boldsymbol{u}}_{\eta}(t)\right) d t \rightarrow \int_{0}^{T} j(\dot{\boldsymbol{u}}(t)) d t .
\end{gathered}
$$

Now, if we pass to the lower limit with $\eta \rightarrow 0$ in (5.29), we have: $\forall \boldsymbol{v} \in$ $L^{2}(0, T ; V)$

$$
\begin{gathered}
\int_{0}^{T}\left((\ddot{\boldsymbol{u}}(t), \boldsymbol{v}(t))+a(\boldsymbol{u}(t), \boldsymbol{v}(t))+\left\langle\Theta^{*} B^{-1} \Theta \boldsymbol{u}(t), \boldsymbol{v}(t)\right\rangle_{V^{\prime}, V}\right) d t \\
-\int_{0}^{T}\left(\langle\boldsymbol{h}(t), \boldsymbol{v}(t)-\dot{\boldsymbol{u}}(t)\rangle_{V^{\prime}, V}+j(\boldsymbol{v}(t))-j(\dot{\boldsymbol{u}}(t))\right) d t
\end{gathered}
$$




$$
\begin{aligned}
\geq & \frac{1}{2}\left(|\dot{\boldsymbol{u}}(T)|^{2}-\left|\boldsymbol{v}_{0}\right|^{2}+a(\boldsymbol{u}(T), \boldsymbol{u}(T))-a\left(\boldsymbol{u}_{0}, \boldsymbol{u}_{0}\right)\right. \\
& \left.+\left\langle\Theta^{*} B^{-1} \Theta \boldsymbol{u}(T), \boldsymbol{u}(T)\right\rangle_{V^{\prime}, V}-\left\langle\Theta^{*} B^{-1} \Theta \boldsymbol{u}_{0}, \boldsymbol{u}_{0}\right\rangle_{V^{\prime}, V}\right) \\
= & \int_{0}^{T}\left(\left(\ddot{\boldsymbol{u}}(t),(\dot{\boldsymbol{u}}(t))+a\left(\boldsymbol{u}(t),(\dot{\boldsymbol{u}}(t))+\left\langle\Theta^{*} B^{-1} \Theta \boldsymbol{u}(t), \dot{\boldsymbol{u}}(t)\right\rangle_{V^{\prime}, V}\right) d t\right.\right.
\end{aligned}
$$

From the last inequality and uniqueness result we deduce that $\boldsymbol{u}$ is the unique solution of the variational problem (5.10) with the conditions (5.7).

Finally, we define $\varphi$ by (5.11) and taking in mind Lemma 5.1, it is now easy to show that $(\boldsymbol{u}, \varphi)$, the unique solution of Problem $P_{V}$, is a limit when $\eta$ converge to 0 in the appropriated paces of $\left(\boldsymbol{u}_{\eta}, \varphi_{\eta}\right)$ the unique solution of Problem $P_{V}^{\eta}$.

\section{REFERENCES}

[1] R. C. Batra and J. S. Yang, Saint-Venant's principle in linear piezoelectricity, Journal of Elasticity 38 (1995), 209-218.

[2] P. Bisenga, F. Lebon and F. Maceri, The unilateral frictional contact of a piezoelectric body with a rigid support, in Contact Mechanics, J.A.C. Martins and Manuel D.P. Monteiro Marques (Eds.), Kluwer, Dordrecht, 2002, p. 347-354.

[3] G. Duvaut, J. L. Lions, Les inéquations en mécanique et en physique, Dunod, Paris, 1972.

[4] C. Eck, J. Jarušek and M. Krbec, Unilateral Contact Problems, Pure and Applied Mathematics 270, Chapman \& Hall/CRC Press, Boca Raton, Florida, 2005.

[5] El H. Essoufi and M. Kabbaj, Existence of solutions of a dynamic Signorini's Problem with non local friction for viscoelastic Piezoelectric Materials, Bull. Math. Soc. SC. Math. Roumanie 48 (2005), 181-195.

[6] El H. Essoufi and M. Sofonea, A Piezoelectric Contact Problem with Slip Dependent Coefficient of friction, Mathematical Modelling and Analysis 9(2004), 229-242.

[7] El H. Essoufi and M. Sofonea, Quasistatic Frictional Contact of a Viscoelastic Piezoelectric Body, Advances in Mathematical Sciences and Applications 14 (2004), 613-631.

[8] W. Han and M. Sofonea, Evolutionary Variational inequalities arising in viscoelastic contact problems, SIAM Journal of Numerical Analysis 38 (2000), 556-579.

[9] W. Han and M. Sofonea, Quasistatic Contact Problems in Viscoelasticity and Viscoplasticity, Studies in Advanced Mathematics 30, Americal Mathematical SocietyIntl. Press, 2002.

[10] T. Ikeda, Fundamentals of Piezoelectricity, Oxford University Press, Oxford, 1990.

[11] I. R. Ionescu, Viscosity solutions for dynamic problems with slip-rate dependent friction, Quart. Appl. Math. I.X 3 (2002), 461-476.

[12] I. R. Ionescu, Q-L. Nguyen, S. Wolf, Slip-dependent friction in dynamic elasticity, Nonlinear Analysis 53 (2003), 375-390.

[13] K. L. Kuttler and M. Shillor, Dynamic contact with Signorini's condition and slip rate dependent friction, Electronic J. Diff. Eqns. 83 (2004), 1-21.

[14] K. L. Kuttler and M. Shillor, Dynamic contact with normal compliance wear and discontinuous friction coefficient, SIAM J. Math. Anal. 34 (2002), 1-27.

[15] F. Maceri and P. Bisegna, The unilateral frictionless contact of a piezoelectric body with a rigid support, Math. Comp. Modelling 28 (1998), 19-28.

[16] R. D. Mindlin, Polarisation gradient in elastic dielectrics, Int. J. Solids Structures 4 (1968), 637-663. 
[17] R. D. Mindlin, Continuum and lattice theories of influence of electromechanical coupling on capacitance of thin dielectric films, Int. J. Solids Structures 4 (1969), 11971213.

[18] R. D. Mindlin, Elasticity, piezoelasticity and crystal lattice dynamics, Journal of Elasticity 4 (1972), 217-280.

[19] J. Nečas and I. Hlaváček, Mathematical Theory of Elastic and Elastico-Plastic Bodies: An Introduction, Elsevier Scientific Publishing Company, Amsterdam, Oxford, New York, 1981.

[20] B. Tengiz and G. Tengiz, Some Dynamic Problems of the Theory of electroelasticity, Memoirs on Differential Equations and Mathematical Physics 10 (1997), 1-53.

[21] R. A. Toupin, The elastic dielectrics, J. Rat. Mech. Analysis 5 (1956), 849-915.

[22] R. A. Toupin, A dynamical theory of elastic dielectrics, Int. J. Engrg. Sci. 1 (1963), 101-126.

M. Kabbaj

Département de Mathématiques

Faculté des Sciences et Techniques

Université Moulay Ismail

B.P. 509-Boutalamine 52000, Errachidia

Maroc

E-mail: kabbaj.mostafa@gmail.com

El-H. Essoufi

Département de Mathématiques

Faculté des Sciences et Techniques

Université Hassan Premier

Km 3, route Casablanca, B.P. 577, Settat

Maroc

E-mail: essoufi@gmail.com

Received: 19.3.2007.

Revised: 15.9.2007. 